\title{
Pareto Boundary of Utility Sets for Multiuser Wireless Systems
}

\author{
Holger Boche, Senior Member, IEEE, Siddharth Naik, and Martin Schubert, Member, IEEE
}

\begin{abstract}
Pareto optimality is an important property in game theory and mechanism design, which can be utilized to design resource allocation strategies in wireless systems. We analyze the structure of the boundary points of certain utility sets based on interference functions. We particularly investigate the cases with no power constraints, with individual power constraints, and with a total power constraint. We display the dependency between Pareto optimality and interference coupling in wireless systems. An axiomatic framework of interference functions and a global dependency matrix is used to characterize interference coupling in wireless systems. The relationship between interference-balancing functions and Pareto optimality of the boundary points is elucidated. Among other results, it is shown that the boundary points of utility sets with individual power constraints and with strictly monotonic interference functions are Pareto-optimal if and only if the corresponding restricted global dependency matrix is irreducible. The obtained results provide certain insight when suitable algorithms can be designed for network utility maximization.
\end{abstract}

Index Terms-Boundary points, feasible utility sets, interference-coupled wireless systems, Pareto optimality.

\section{INTRODUCTION}

$\mathbf{P}$ ERFORMANCE tradeoffs in multiuser systems occur when users share a common resource or if they are coupled by mutual interference. This is typical for wireless systems and also for certain wireline connections, e.g., twisted-pair copper wires used for DSL transmission. Such tradeoffs are commonly characterized by the utility set, sometimes referred to as the utility region or quality-of-service (QoS) region. The utility region $\mathbf{U}$ is defined as the set of all achievable utility vectors $\boldsymbol{u}=\left[u_{1}, \ldots, u_{K}\right]^{T}$, where $K \geq 2$ is the number of users, $\boldsymbol{u} \in \mathbf{U}$. The task of resource allocation crucially depends on the properties of $\mathbf{U}$. It especially depends on the boundary points since the operating points of resource allocation strategies usually are on the boundary. We investigate the structure of the Pareto-optimal boundary points of utility sets, which are frequently encountered in wireless systems.

A thorough understanding of the Pareto-optimal boundary points is an important basis for many practical and theoretical

Manuscript received February 03, 2009; revised November 11, 2009 and June 29, 2010; accepted August 31, 2010; approved by IEEE/ACM TRANSACTIONS ON NETWORKING Editor S. Sarkar. Date of publication November 15, 2010; date of current version April 15, 2011.

H. Boche and S. Naik are with the Technical University of Berlin, Heinrich Hertz Institute, 10587 Berlin, Germany (e-mail: holger.boche@ mk.tu-berlin.de; naik@hhi.fraunhofer.de).

M. Schubert is with the German Sino Lab for Mobile Communications, Heinrich Hertz Institute, 10587 Berlin (e-mail: schubert@hhi.fraunhofer.de).

Digital Object Identifier 10.1109/TNET.2010.2083683 concepts such as network utility maximization, game theory, axiomatic bargaining theory, and mechanism design [1]. For example, a classical result from mechanism design is that there exists no social choice function, which simultaneously satisfies the axioms of efficiency, strategy proofness, and nondictatorship for systems with two or more users and two or more resources [2], [3]. In [2] and [3], Pareto optimality implies efficiency, and strategy proofness represents the property that a resource allocation strategy cannot be manipulated by any user, i.e., a user has no incentive to misrepresent its utility to a central controller in a decentralized system. Pareto optimality provides the system designer a tool to check for nonmanipulation of the resource allocation strategy (strategy proofness).

Pareto optimality depends on the interference coupling in the system and on power constraints. Under further restrictions on the interference functions, e.g., the linear interference functions frequently encountered in wireless systems, Pareto optimality of the boundary points is a necessary condition to obtain strict convexity of the region. In the case of total power constraints, Pareto optimality of the boundary points is a necessary and sufficient condition for the strict convexity of the region. This connection, particularly that of strict convexity of the region has been analyzed in [4] and [5]. As can be seen, there exists a connection between the investigation of Pareto-optimal boundary points and investigation of the convexity properties of the region. Convexity can help to design at least numerically simple algorithmic solutions to resource allocation problems for utility sets. Furthermore, Pareto optimality of boundary points implies there is always an inherent tradeoff between the performance of the users if we would like to allocate resources at these Pareto-optimal boundary points.

An axiomatic approach to interference functions was proposed by Yates in [6] with extensions in [7] and [8]. The Yates framework of standard interference functions is general enough to incorporate cross-layer effects, and it serves as a theoretical basis for a variety of algorithms. Certain examples include: beamforming [9], code division multiple access (CDMA) [10], [11], base station assignment, robust design, and networking [12]. The framework can be used to combine power control and adaptive receiver strategies. Certain examples, where this has been successfully achieved are as follows. In [13], it was proposed to incorporate admission control to avoid unfavorable interference scenarios. In [14], it was proposed to adapt the QoS requirements to certain network conditions. In [15], a power control algorithm using fixed-point iterations was proposed for a modified cost function, which permits control of convergence behavior by adjusting fixed weighting parameters. The Yates framework under certain 
natural assumptions is equivalent to the general interference function framework [16] (explained in Appendix C). The Yates framework has power control as its main application and is useful in achieving a particular point, e.g., iterative convergence of the distributed power update rule.

The general interference function framework [16] inherits these properties and, being more general, can be utilized beyond power control problems. The general interference function framework is particularly suited to analyzing regions and is one of the main reasons why we use it to investigate Pareto-optimal boundary points of regions. Such a framework is not limited to cellular wireless networks. In a general multipoint-to-multipoint system, all users interfere with every other user. Related work for the special case of log-convex interference functions was considered in [17]. In our paper, we significantly extend these results and utilize another technique to analyze the broader class of general interference functions, where log-convex interference functions are a subclass. We elucidate the connection between the interference function framework and the domain of feasible utility sets through certain intuitive and natural properties of interference functions encountered frequently in wireless systems. The contributions of the paper are as follows.

- In Section III, we characterize the structure of the boundary points of utility regions without power constraints and develop certain useful analytical tools, which are useful in investigating the other cases.

- In Section IV, we characterize necessary and sufficient conditions for the boundary points of utility sets with individual power constraints to be Pareto-optimal.

- In Section V, we characterize the structure of the boundary points of utility regions with a total power constraint. We extend the results of [17], omitting the requirement of logconvex interference functions.

- In Section VI, we characterize the boundary of these utility sets in terms of the interference-balancing functions. With the help of this analysis, we are in a position to comment on the characteristics of certain power-constrained utility sets either in terms of corresponding interference functions or interference-balancing functions, which is a useful tool for further analysis in multiuser wireless systems.

\section{INTERFERENCE-COUPLED WIRELESS SYSTEMS}

In this paper, we shall investigate the case of interference-coupled wireless systems. Before we begin to describe our system model and present the relevant definitions, we provide certain notational conventions used in the paper in Section II-A.

\section{A. Preliminaries and Notation}

Matrices and vectors are denoted by bold capital letters and bold lowercase letters, respectively. Let $\boldsymbol{y}$ be a vector, then $y_{\boldsymbol{l}}=$ $[\boldsymbol{y}]_{l}$ is the $l$ th component. Likewise, $G_{m n}=[G]_{m n}$ is a component of the matrix $\boldsymbol{G}$. Let $\|\boldsymbol{y}\|_{\infty}=\max _{1 \leq l \leq K} y_{l}$, where $\boldsymbol{y}$ has $K$ elements. The notation $\boldsymbol{y} \geq 0$ implies that $y_{l} \geq 0$ for all components $l . x \supsetneqq y$ implies component-wise inequality with strict inequality for at least one component. Similar definitions hold for the reverse directions. Let $\mathbf{X}$ denote a set, and $\partial \mathbf{X}$ the boundary of the set $\mathbf{X}$. Finally, $\boldsymbol{x} \neq \boldsymbol{y}$ implies that the vector differs in at least one component. The set of nonnegative real numbers is denoted as $\mathbb{R}_{+}$. The set of positive real numbers is denoted as $\mathbb{R}_{++}$. Let $\underline{\boldsymbol{e}}(r)$ be a $(K+1)$-dimensional vector, which is the all-zero vector with the $r$ th component set to one, i.e.,

$$
[\underline{\boldsymbol{e}}(r)]_{k}=\left\{\begin{array}{ll}
1, & k=r \\
0, & k \neq r
\end{array} .\right.
$$

\section{B. Interference Coupling}

The signal-to-interference-plus-noise ratio (SINR) is an important measure for user performance in wireless systems. Many other performance measures have a direct relationship with SINR (refer to Section II-C for examples). Consider $K$ users with transmit powers $\boldsymbol{p}=\left[p_{1}, \ldots, p_{K}\right]^{T}$ and $\mathcal{K}:=\{1, \ldots, K\}$. The noise power at each receiver is $\sigma^{2}$. Hence, the SINR at each receiver depends on the extended power vector

$$
\underline{\boldsymbol{p}}=\left[\begin{array}{c}
\boldsymbol{p} \\
\sigma^{2}
\end{array}\right]=\left[p_{1}, \ldots, p_{K}, \sigma^{2}\right]^{T} .
$$

The resulting SINR of user $k$ is $\operatorname{SINR}_{k}(\boldsymbol{p})=p_{k} / \mathcal{I}_{k}(\boldsymbol{p})$, where $\mathcal{I}_{k}$ is the interference (plus noise) as a function of $\boldsymbol{p}$. In order to model interference, we shall follow the axiomatic approach proposed in [6] and [11], explained in detail in Appendix C. The structure of the SINR region depends on the interference coupling in the system. We can define the system as coupled as follows. For given $k, r$, we define

$$
\left[\boldsymbol{D}_{\mathcal{I}}(\boldsymbol{p})\right]_{k r}=\left\{\begin{aligned}
& 1, \quad \text { if } \exists \delta_{r}(\boldsymbol{p})>0 \text { such that } \\
& f_{r}(\delta, \boldsymbol{p})=\mathcal{I}_{k}(\underline{\boldsymbol{p}}-\delta \underline{\boldsymbol{e}}(r)) \\
& \text { is strictly monotone decreasing } \\
& \text { for } 0 \leq \delta \leq \delta_{r}(\boldsymbol{p}) \\
& 0, \quad \text { otherwise }
\end{aligned}\right.
$$

This condition can be further relaxed as follows. Instead of requiring the above property for a specific power vector $\boldsymbol{p}$, we now define the system as "coupled" if there is some arbitrary power vector $\boldsymbol{p}$ such that the matrix $\left[\boldsymbol{D}_{\mathcal{I}}(\boldsymbol{p})\right]_{k r}=1$. Thereby, we obtain the global dependency matrix, which is independent of the choice of power vector $\boldsymbol{p}$.

Definition 1: Global dependency matrix: $\boldsymbol{G}_{\mathcal{I}}$ is the global dependency matrix, given by

$$
\left[\boldsymbol{G}_{\mathcal{I}}\right]_{k r}=\left\{\begin{array}{cc}
1, & \text { if there exists a } \boldsymbol{p} \geq \mathbf{0} \text { such that } \\
& {\left[\boldsymbol{D}_{\mathcal{I}}(\boldsymbol{p})\right]_{k r}>0} \\
0, & \text { otherwise }
\end{array} .\right.
$$

Remark 1: We denote the matrix $\boldsymbol{G}_{\mathcal{I}}=: \boldsymbol{G}$. We have denoted it initially as $\boldsymbol{G}_{\mathcal{I}}$ in Definition 1 to display the interconnection with the interference functions.

The nonzero entries in $\boldsymbol{G}$ represent the transmitter/receiver pairs, which are coupled by interference. Note that this coupling model includes the widely used concept of a "link gain matrix" as a special case. An intuitive interpretation of the dependency matrix and the global dependency matrix is as follows: A zero entry in row $k$ and column $r$ implies that no interference is received by a user $k$ from user $r$, irrespective of how large the transmission power of user $r$ is. As an example, consider that users are assigned to different orthogonal resources separated by adaptive interference rejection techniques. An example due 
to natural causes, where a user $r$ might not cause interference to another user $k$, is when user $r$ experiences shadowing. In the remainder of this paper, we shall use $\boldsymbol{G}$ in order to analyze the effects of interference coupling on the structure of the boundary. We assume that $\boldsymbol{G}$ is an irreducible matrix [18, pp. 360-361] (see also the standard reference for nonnegative matrices [19]). This implies that each user is interfered by at least one other user. Irreducibility of $\boldsymbol{G}$ is equivalent to strong connectivity of the graph $\mathcal{G}(\boldsymbol{G})$, which is defined to be the directed graph of $\mathcal{K}=\{1, \ldots, K\}$ nodes, in which there is a directed edge (or link) leading from node $r \in \mathcal{K}$ to $k \in \mathcal{K}$ if and only if $[G]_{k r}>0$. The matrix $\boldsymbol{G}$ is called the adjacency matrix of the graph $\mathcal{G}(\boldsymbol{G})$. This graph is said to be strongly connected if, for each pair of nodes $(k, r)$, there is an uninterrupted sequence of directed edges leading from $r$ to $k$. Note that the direction matters in the definition of strong connectivity for directed graphs. The following proposition is a standard result [18, p. 362, Th. 6.2.24]. Matrix $G$ is irreducible if and only if $\mathcal{G}(\boldsymbol{G})$ is strongly connected.

We now introduce certain important properties of interference functions, namely strict monotonicity, strict positivity, and the dependency set. These properties will be needed later in the analysis.

Definition 2: Dependency set. Based on the global dependency matrix as defined by (2), we say that $L_{k}$ is the dependency set of user $k$ if

$$
L_{k}=\left\{r \in \mathcal{K} \mid[\boldsymbol{G}]_{k r}=1\right\} .
$$

Remark 2: We assume that the dependency set is nonempty throughout the paper.

This is the set of transmitters, which have an impact on user $k$. Our framework allows for systems, where each user experiences interference from at least one other user. An example of the dependency set when all users interfere with each other is given by $L_{k}=\{j \in \mathcal{K} \mid j \neq k\}$ for the case of no self-interference. For the case with self-interference, an example of the dependency set is $L_{k}=\mathcal{K}$.

Definition 3: Strict monotonicity: $\mathcal{I}_{k}(\boldsymbol{p})$ is said to be strictly monotonic on its respective dependency set $L_{k}$ if $\boldsymbol{p}^{(1)} \geq \boldsymbol{p}^{(2)}$ with $p_{r}^{(1)}>p_{r}^{(2)}$ for some $r \in L_{k}$, implies $\mathcal{I}_{k}\left(\boldsymbol{p}^{(1)}\right)>\mathcal{I}_{k}\left(\boldsymbol{p}^{(2)}\right)$.

In other words, $\mathcal{I}_{k}(\underline{p})$ is strictly increasing in at least one of the first $K$ components. At certain times, we shall define our domain for interference functions as $\mathbb{R}_{+}^{K}$, instead of $\mathbb{R}_{+}^{K+1}$, e.g., in Definition 4 to follow. However, we usually imply the domain of the interference function to be $\mathbb{R}_{+}^{K+1}$ unless otherwise specified.

Definition 4: Strict positivity: An interference function $\mathcal{I}_{k}(\boldsymbol{p})$ is said to be strictly positive on the dependency set $L_{k}$ if, for any $\boldsymbol{p} \geq \mathbf{0}$, with $\boldsymbol{p} \neq[0, \ldots, 0]^{T}$ and $p_{l}>0$ for $l \in L_{k}$, we have that $\mathcal{I}(\boldsymbol{p})>0$.

Remark 3: Throughout the paper, the following convention is used.

- If $\mathcal{I}(\boldsymbol{p})$ is utilized, it means that there is no noise and we consider only axioms $A 1$ to $A 3$.

- If $\mathcal{I}(\underline{p})$ is utilized, it means that there is noise and we consider axioms $A 1$ to $A 4$.

We have $\mathcal{I}(\boldsymbol{p})>0$ in the presence of noise. However, in Definition 4 we check for positivity of the interference function with respect to the power vector independent of the noise. $\mathcal{I}_{1}, \ldots, \mathcal{I}_{K}$ are strictly positive on their dependency sets $L_{1}, \ldots, L_{K}$, respectively. From a practical point of view, strict positivity on the dependency set seems very natural in wireless systems. Nonetheless, it is an important mathematical restriction whose impact shall be noticed in Section III while proving the desired results. Strict positivity of the interference function on the dependency set is a stronger condition than $A 1$ (refer to Appendix C).

Example 1: Consider the following two examples.

1) $\mathcal{I}(\boldsymbol{p})=\sum_{k=1}^{K} v_{k} p_{k}, \boldsymbol{v}>\mathbf{0}, \boldsymbol{v} \neq[0, \ldots, 0]^{T}$ is a strictly positive interference function on the dependency set.

2) $\mathcal{I}(\boldsymbol{p})=\prod_{k} p_{k}^{\omega_{k}}, \boldsymbol{\omega} \geq \mathbf{0}, \sum_{k=1}^{K} \omega_{k}=1$ is not a strictly positive interference function on the dependency set.

\section{Utility Sets Based on SINR}

Until now, we have focused on interference coupling aspects, where interference is a function of the powers of the various users and noise. We shall now analyze the resulting utility set. In this paper, "utility" can represent a certain arbitrary performance measure, which depends on the SINR by a strictly monotonic and continuous function $\phi$ defined on $\mathbb{R}_{+}$. The utility of user $k$ is

$$
u_{k}(\underline{\boldsymbol{p}})=\phi_{k}\left(\operatorname{SINR}_{k}(\underline{\boldsymbol{p}})\right), \quad k \in \mathcal{K} .
$$

An example of the above case is capacity: $\phi(x)=\log (1+x)$. Related performance indicators when we would like to minimize the objective function, which is a function of SINR, are: 1) minimum mean square error (MMSE): $\phi(x)=1 /(1+x)$; 2) BER: $\phi(x)=Q(\sqrt{x})$; or 3$)$ high-SINR approximation of BER: $\phi(x)=x^{-\alpha}$ with diversity order $\alpha$. We shall analyze the Pareto-optimal boundary of certain sets for the following cases:

1) utility sets without power constraints;

2) utility sets with individual power constraints;

3) utility sets with total power constraints.

\section{ANALYSIS OF THE PARETO-OPtIMAL Boundary OF UTILITY SETS WithOUT POWER CONSTRAINTS}

In this section, we shall analyze the properties of boundary points of utility sets without power constraints.

Remark 4: In this case, we have the following power vector $\boldsymbol{p} \in \mathcal{P}$, here $\mathcal{P}=\mathbb{R}_{+}^{K}$, i.e., we have the case of interference limited systems.

Analyzing the case in the high SINR regime helps to clearly bring out the effects of interference coupling. Section III-A will outline the structure of the QoS region without power constraints and introduce weak Pareto optimality and Pareto optimality of boundary points, which shall be used here and in Section IV. The analytical results, methodology, and proof technique developed in Section III-B is utilized to obtain results also in Section IV-B.

\section{A. Structure of the QoS Region Without Power Constraints}

Consider the SIR (since we have no noise, we use SIR and not SINR) feasible region for users $\mathcal{K}=\{1, \ldots, K\}$, without power constraints, which is defined as the sublevel set

$$
\mathcal{S}(\mathcal{I})=\left\{\boldsymbol{\gamma} \in \mathbb{R}_{+}^{K} \mid C(\boldsymbol{\gamma}, \mathcal{I}) \leq 1\right\}
$$


where $\boldsymbol{\gamma}=\left[\gamma_{1}, \ldots, \gamma_{K}\right]^{T}$ is a vector of SIR values, whose feasibility is determined by the min-max optimum (see, e.g., [20] and [21]) and $\boldsymbol{\gamma}=\left[\gamma_{1}, \ldots, \gamma_{K}\right]^{T}$, and $\mathcal{I}=\left[\mathcal{I}_{1}, \ldots, \mathcal{I}_{K}\right]$ is a vector of interference functions. We introduce the interference-balancing function as follows:

$$
C(\boldsymbol{\gamma}, \mathcal{I})=\inf _{\boldsymbol{p}>\mathbf{0}} \max _{k \in \mathcal{K}} \frac{\gamma_{k} \mathcal{I}_{k}(\boldsymbol{p})}{p_{k}} .
$$

The structure of the SIR set $\mathcal{S}(\mathcal{I})$ depends on the properties of interference-balancing function $C(\boldsymbol{\gamma}, \mathcal{I})$, which in turn depends on the properties of the underlying interference functions $\mathcal{I}_{1}, \ldots, \mathcal{I}_{K}$. Let $\gamma_{k}$ be the inverse function of $\phi_{k}$, then $\gamma_{k}\left(u_{k}\right)$ is the minimum SIR level needed by the $k$ th user to satisfy the QoS target $u_{k}$. Let $\boldsymbol{u} \in \mathbf{U}$ be a vector of QoS values, then the associated SIR vector is

$$
\gamma(\boldsymbol{u})=\left[\gamma_{1}\left(u_{1}\right), \ldots, \gamma_{K}\left(u_{K}\right)\right]^{T} .
$$

QoS values $\boldsymbol{u} \in \mathbf{U}$ are feasible if and only if the interferencebalancing function $C(\boldsymbol{\gamma}(\boldsymbol{u}), \mathcal{I}) \leq 1$. The QoS feasible set is the sublevel set

$$
\mathbf{U}=\{\boldsymbol{u}: C(\gamma(\boldsymbol{u}), \mathcal{I}) \leq 1\} .
$$

We are specifically interested in the boundary of $\mathbf{U}$, which is characterized by $C(\boldsymbol{\gamma}(\boldsymbol{u}), \mathcal{I})=1$. We recollect that the boundary is denoted as $\partial \mathbf{U}$. We shall now describe what we mean by weak Pareto-optimal and Pareto-optimal boundary points, respectively.

Definition 5: A point $\boldsymbol{u} \in \partial \mathbf{U}$ is weak Pareto-optimal if there is no $\hat{\boldsymbol{u}} \in \mathbf{U}$ with $\hat{\boldsymbol{u}}>\boldsymbol{u}$.

From a practical point of view, this implies that it is not possible to collectively improve the performance of all the users in the system.

Definition 6: A boundary point $\boldsymbol{u} \in \partial \mathbf{U}$ is Pareto-optimal if there is no $\hat{\boldsymbol{u}} \in \mathbf{U}$ with $\hat{\boldsymbol{u}} \supsetneqq \boldsymbol{u}$.

From a practical point of view, this implies that it is not possible to improve the performance of one user without simultaneously decreasing the performance of another user.

Lemma 1: A boundary point $\boldsymbol{u} \in \partial \mathbf{U}$ is Pareto-optimal if and only if $\boldsymbol{\gamma}(\boldsymbol{u}) \in \partial \mathcal{S}(\mathcal{I})$ is Pareto-optimal [17].

Proof: This is a direct consequence of the strict monotonicity of the mapping in (4). Pareto-optimal points in $\mathcal{S}$ are mapped to Pareto-optimal points in $\mathbf{U}$, and vice versa. Weak Pareto boundary segments in $\mathcal{S}$ are parallel to the coordinate axes. These segments are mapped to parallel segments in $\mathbf{U}$, and vice versa.

From a wireless communications perspective (physical-layer perspective), choosing a Pareto-optimal boundary point in the feasible utility region implies choosing an efficient operating point. In social choice theory, a social choice function (SCF) $f$ is said to be efficient if, $\forall \boldsymbol{u} \in \mathcal{U}^{K}$, there is no power allocation $\boldsymbol{p} \in \mathcal{P}$ such that $u_{k}\left(\boldsymbol{p}^{(k)}\right) \geq u_{k}\left(f_{k}(\boldsymbol{u})\right)$, for all users $k \in \mathcal{K}$, and $u_{k}\left(\boldsymbol{r}^{(k)}\right)>u_{k}\left(f_{k}(\boldsymbol{u})\right)$ for some user $k \in \mathcal{K}$ [22]. An example of such a utility could be SIR $\boldsymbol{\gamma}$.

From Lemma 1, we know that for any utility set according to the above definition, we can analyze Pareto optimality by focusing on the underlying SIR set. The results transfer automati- cally to the corresponding utility sets, where utility depends on the SIR according to (4).

\section{B. Pareto-Optimal Boundary of Utility Sets Without Power Constraints}

This section presents the main results in relation to boundary points of utility sets without power constraints. The results presented here will also be later used in Section III-B, and the proof technique of Theorem 1 will be used as foundation for further research work. We begin by presenting certain preliminary results as a prelude to Theorem 1 .

Lemma 2: Let $\mathcal{I}_{1}, \ldots, \mathcal{I}_{K}$ be strictly positive interference functions on their respective dependency sets $L_{k}$. Let $\mathcal{I}_{1}, \ldots, \mathcal{I}_{K}$ be strictly monotonic on their respective dependency sets $L_{k}$. Let there be a sequence of power vectors $\left\{\boldsymbol{p}^{(n)}\right\}_{n \in \mathbb{N}}$, which can be decreased as follows:

$$
\lim _{n \rightarrow \infty} \mathcal{I}_{k}\left(\boldsymbol{p}^{(n)}\right)=0 .
$$

Then, for all users $j$, with $j \in L_{k}$, we have that

$$
\lim _{n \rightarrow \infty} p_{j}^{(n)}=0 .
$$

Therefore, from (10), we have that

$$
\lim _{n \rightarrow \infty} \max _{j \in L_{k}} p_{j}^{(n)}=0 .
$$

Proof: It is clear that (10) implies (11). For the sake of obtaining a contradiction, let us assume that (10) is not true. Therefore, there exists a $k_{0} \in L_{k}$ such that $\lim _{\sup _{n \rightarrow \infty}} p_{k_{0}}^{(n)}=$ $c_{1}>0$. Then, there must exist a subsequence $\left\{n_{l}\right\}_{l \in \mathbb{N}}$ of natural numbers such that $p_{k_{0}}^{\left(n_{l}\right)} \geq c_{1} / 2>0$ for all $l \in \mathbb{N}$. Thus, for all $l \in \mathbb{N}$ due to strict monotonicity and strict positivity, we have that $\mathcal{I}\left(\boldsymbol{p}^{\left(n_{l}\right)}\right) \geq \mathcal{I}\left(c_{1} \boldsymbol{e}\left(k_{0}\right)\right) / 2=c_{1} \mathcal{I}\left(\boldsymbol{e}\left(k_{0}\right)\right) / 2=c_{2}>0$. Therefore, we have that $\liminf _{l \rightarrow \infty} \mathcal{I}\left(\boldsymbol{p}^{\left(n_{l}\right)}\right) \geq c_{2}>0$, which is in contradiction of (9).

Remark 5: The decisive property used to obtain the above result was the strict positivity of the interference functions.

We now present two results in relation to the interferencebalancing function $C(\boldsymbol{\gamma}, \mathcal{I})$. We shall see these two results used later in the proof of Theorem 2.

Lemma 3: Let $\mathcal{I}_{1}, \ldots, \mathcal{I}_{K}$ be strictly positive interference functions on their respective dependency sets. Then, for all $\gamma_{1}, \ldots, \gamma_{K}>0$, we have that

$$
C(\boldsymbol{\gamma}, \mathcal{I})=\inf _{\boldsymbol{p}>\mathbf{0}} \max _{k \in \mathcal{K}} \frac{\gamma_{k} \mathcal{I}_{k}(\boldsymbol{p})}{p_{k}}>0 .
$$

Proof: The proof is given in Appendix A.

The next result, Lemma 4, is an example of an impossibility result, which states the following. Let us perform interference balancing for a subset of users $\mathcal{K}^{(1)}$ or for a subset of users $\mathcal{K}^{(2)}$, where $\mathcal{K}^{(1)} \cap \mathcal{K}^{(2)}=\emptyset$ and $\mathcal{K}^{(1)} \cap \mathcal{K}^{(2)}=\mathcal{K}$. The interference balancing is performed by switching off the users in the other set. Then, it is not possible to perform better than when all the $K$ users are active.

To present this result, we introduce the following notation. Let $\mathbb{R}_{+, 0}^{K, k_{1}}$ be the set of all vectors $p \in \mathbb{R}_{+}^{K}$ with power vector $\boldsymbol{p}=\left[0, \ldots, 0, p_{k_{1}+1}, \ldots, p_{K}\right]^{T}$ and $p_{l}>0$, for $k_{1}+1 \leq l \leq$ 
$K, 0<k_{1}<K$. Here, $k_{1}$ is an arbitrary number, i.e., the two subsets of users $\mathcal{K}^{(1)}:=\left\{1, \ldots, k_{1}\right\}$ and $\mathcal{K}^{(2)}:=\left\{k_{1}+\right.$ $1, \ldots, K\}$ are an arbitrary disjoint division of the users in the set $\mathcal{K}$, i.e., $\mathcal{K}^{(1)} \cap \mathcal{K}^{(2)}=\emptyset$.

We know that $\mathcal{I}_{k}(\boldsymbol{p})$ is a monotonic increasing function with respect to $\boldsymbol{p}$ ( $A 3$, see Definition 8 ). Then, for power vectors $\boldsymbol{p} \in \mathbb{R}_{+, 0}^{K, k_{1}}$ and $\tilde{\boldsymbol{p}} \in \mathbb{R}_{++}^{K}$, we have the following inequality: $\inf _{\boldsymbol{p} \in \mathbb{R}_{+, 0}^{K, k_{1}}} \mathcal{I}_{k}(\boldsymbol{p}) \leq \inf _{\tilde{\boldsymbol{p}} \in \mathbb{R}_{++}^{K}} \mathcal{I}_{k}(\tilde{\boldsymbol{p}})$. Hence, we have the following expression:

$$
\inf _{\boldsymbol{p} \in \mathbb{R}_{++}^{K}} \max _{k_{1}<k \leq K} \frac{\gamma_{k} \mathcal{I}_{k}(\boldsymbol{p})}{p_{k}} \geq \inf _{\boldsymbol{p} \in \mathbb{R}_{+, 0}^{K, k_{1}}} \max _{k_{1}<k \leq K} \frac{\gamma_{k} \mathcal{I}_{k}(\boldsymbol{p})}{p_{k}}
$$

In Lemma 3 and throughout the paper, $\boldsymbol{\gamma}^{(1)}$ and $\mathcal{I}^{(1)}$ have dimensions $k_{1}$ and $\boldsymbol{\gamma}^{(2)}$ and $\mathcal{I}^{(2)}$ has dimensions $K-k_{1}$, where $\mathcal{I}^{(1)}: \mathcal{P} \mapsto \mathbb{R}_{+}^{k_{1}}$, with $\boldsymbol{p} \in \mathcal{P} \subset \mathbb{R}_{+}^{k_{1}}$ and $\mathcal{I}^{(2)}: \mathcal{P} \mapsto \mathbb{R}_{+}^{K-k_{1}}$, with $\boldsymbol{p} \in \mathcal{P} \subset \mathbb{R}_{+}^{K-k_{1}} . \mathcal{I}^{(1)}$ and $\mathcal{I}^{(2)}$ are tuples of interference functions. If we have a set $\mathcal{K}=\{1, \ldots, K\}$, we can always split this set into two separate disjoint sets in the case without power constraint.

The users in the set $\mathcal{K}^{(1)}$ do not see the users in the set $\mathcal{K}^{(2)}$ as interference, and vice versa. This is due to the fact that we fix the powers of the users in the other set to zero, while analyzing a particular set of users.

$\mathcal{I}^{(1)}$ and $\mathcal{I}^{(2)}$ correspond to the vector of interference functions for the sets of users $\mathcal{K}^{(1)}$ and $\mathcal{K}^{(2)}$, respectively.

Lemma 4: The interference-balancing function $C(\gamma, \mathcal{I})$ can be written as

$$
C(\boldsymbol{\gamma}, \mathcal{I})=\max \left(C\left(\boldsymbol{\gamma}^{(1)}, \mathcal{I}^{(1)}\right), C\left(\boldsymbol{\gamma}^{(2)}, \mathcal{I}^{(2)}\right)\right)
$$

where $C\left(\boldsymbol{\gamma}^{(1)}, \mathcal{I}^{(\mathbf{1})}\right)$ are the interference-balancing functions corresponding to a set of users $\left\{1, \ldots, k_{1}\right\}$, and $C\left(\boldsymbol{\gamma}^{(\mathbf{2})}, \mathcal{I}^{(\mathbf{2})}\right)$ are the interference-balancing functions corresponding to a set of users $\left\{k_{1}+1, \ldots, K\right\}$.

Furthermore, we can find a SIR vector $\gamma>0$ such that $C(\boldsymbol{\gamma}, \mathcal{I})=C\left(\boldsymbol{\gamma}^{(1)}, \mathcal{I}^{(1)}\right)$.

Proof: The proof is given in Appendix B.

Lemmas 2-4 assist us in proving the results, which characterize the Pareto-optimal boundary points of feasible utility sets. Equipped with these lemmas, we are now in a position to present the main results of this section. The next theorem will provide the conditions when any $\gamma$ on the boundary can always be achieved with equality, i.e., $\operatorname{SIR}_{k}\left(\boldsymbol{p}^{*}\right)=\gamma_{k}$, for all $k \in \mathcal{K}$. Note that this need not be fulfilled in general. The set defined by (5) corresponds to a SIR set without power constraints. In general, it can be seen that a boundary point can be achieved in an "asymptotic sense." However, the following Theorem 1 states that such a point can always be achieved under the specified conditions.

Theorem 1: Let $\mathcal{I}_{1}, \ldots, \mathcal{I}_{K}$ be strictly positive interference functions on their respective dependency sets. Let $\mathcal{I}_{1}, \ldots, \mathcal{I}_{K}$ be strictly monotonic on their respective dependency sets, and let the coupling matrix $\boldsymbol{G}$ be irreducible. Then, for all $\gamma>\mathbf{0}$, there exists a power vector $\boldsymbol{p}^{*}=\boldsymbol{p}^{*}(\gamma)>\mathbf{0}$ such that

$$
C(\boldsymbol{\gamma}, \mathcal{I}) p_{k}^{*}=\gamma_{k} \mathcal{I}_{k}\left(\boldsymbol{p}^{*}\right) \quad \forall k \in \mathcal{K} .
$$

Proof: Here, we are analyzing the case without power constraints on the users. Hence, there is no noise present. The restriction of $\|\boldsymbol{p}\|_{\infty}=1$ has been introduced for mathematical convenience to obtain compactness of the concerned set. Now, we have that

$$
\begin{aligned}
C(\boldsymbol{\gamma}, \mathcal{I}) & =\inf _{\boldsymbol{p}>\mathbf{0}} \max _{k \in \mathcal{K}} \frac{\gamma_{k} \mathcal{I}_{k}(\boldsymbol{p})}{p_{k}} \\
& \stackrel{(a)}{=} \inf _{\boldsymbol{p}>\mathbf{0},\|\boldsymbol{p}\|_{\infty}=1} \max _{k \in \mathcal{K}} \frac{\gamma_{k} \mathcal{I}_{k}(\boldsymbol{p})}{p_{k}}
\end{aligned}
$$

i.e., the condition $\|p\|_{\infty}=1$ does not alter the problem structure. We obtain (a) from the axiom $A 2$ of scale invariance of interference functions (see Definition 8) as follows. Since we are in the noise-free case, we can scale the power vectors $\lambda \boldsymbol{p}=$ $\left[\lambda p_{1}, \ldots, \lambda p_{K}\right]$. Then, we have the following expression:

$$
\frac{\gamma_{k} \mathcal{I}_{k}(\lambda \boldsymbol{p})}{\lambda p_{k}}=\frac{\gamma_{k} \lambda \mathcal{I}_{k}(\boldsymbol{p})}{\lambda p_{k}}=\frac{\gamma_{k} \mathcal{I}_{k}(\boldsymbol{p})}{p_{k}} .
$$

For all $\epsilon>0$, there exists a vector $\boldsymbol{p}(\epsilon)>\mathbf{0},\|\boldsymbol{p}(\epsilon)\|_{\infty}=1$, with

$$
C(\boldsymbol{\gamma}, \mathcal{I})+\epsilon \geq\left(\frac{\gamma_{k} \mathcal{I}_{k}(\boldsymbol{p}(\epsilon))}{p_{k}(\epsilon)}\right), \quad k \in \mathcal{K}
$$

Next, we show that there exists an $\epsilon_{0}>0$ and $c_{1}>0$ such that for all $0<\epsilon \leq \epsilon_{0}$, we have that

$$
\min _{k \in \mathcal{K}} p_{k}(\epsilon) \geq c_{1}>0
$$

The proof shall be achieved through contradiction. For the sake of obtaining the desired contradiction, let us assume that (13) is not true. Then, there exists a sequence $\left\{\epsilon_{n}\right\}_{n \in \mathbb{N}}$ with $\epsilon_{n+1}<\epsilon_{n}$ and $\lim _{n \rightarrow \infty} \epsilon_{n}=0$, such that $\lim _{n \rightarrow \infty} \min _{k \in \mathcal{K}} p_{k}\left(\epsilon_{n}\right)=0$. Then, there must exist a user $k_{0}$ and a subsequence $\left\{n_{l}\right\}_{l \in \mathbb{N}}$ of natural numbers such that $\lim _{l \rightarrow \infty} p_{k_{0}}\left(\epsilon_{n_{l}}\right)=0$. This follows from the fact that we are distributing an infinite number of natural numbers over a finite set (since there is a finite numbers of users), and hence there exists such a subsequence and the possibility of finding a required index $k_{0} \cdot{ }^{1}$

We shall call $\hat{\epsilon}_{l}:=\epsilon_{n_{l}}$, for $l \in \mathbb{N}$. We know that $0<$ $\gamma_{k_{0}} \mathcal{I}_{k_{0}}\left(\boldsymbol{p}\left(\hat{\epsilon}_{l}\right)\right) \leq\left(C(\boldsymbol{\gamma}, \mathcal{I})+\hat{\epsilon}_{l}\right) p_{k_{0}}\left(\hat{\epsilon}_{l}\right)$. Then, we have that

$$
\lim _{l \rightarrow \infty} \mathcal{I}_{k_{0}}\left(\boldsymbol{p}\left(\hat{\epsilon}_{l}\right)\right)=0 .
$$

The interference function $\mathcal{I}_{k_{0}}$ is strictly positive on its dependency set. Then, from (14), we must have for all users $r \in L_{k_{0}}$ that $\lim _{l \rightarrow \infty} p_{r}\left(\hat{\epsilon}_{l}\right)=0$. However, this leads us to the conclusions that for all users $r \in L_{k_{0}}$, we have $\gamma_{r} \mathcal{I}_{r}\left(\boldsymbol{p}\left(\hat{\epsilon}_{l}\right)\right) \leq$ $\left(C(\boldsymbol{\gamma}, \mathcal{I})+\hat{\epsilon}_{l}\right) p_{r}\left(\hat{\epsilon}_{l}\right)$ and $\lim _{l \rightarrow \infty} \mathcal{I}_{r}\left(\boldsymbol{p}\left(\hat{\epsilon}_{l}\right)\right)=0$. Therefore, for all $n \in L_{r}$ we have that $\lim _{l \rightarrow \infty} p_{n}\left(\hat{\epsilon}_{l}\right)=0$.

Now, we select any $m \in\{1, \ldots, K\}$. Since $G$ is an irreducible matrix, then there exist indices $n_{0}, n_{1}, \ldots, n_{t}$ with $n_{0}=$ $k_{0}, n_{i+1} \in L_{n_{i}}$, and $n_{t}=m$. Here, $L_{n_{i}}$ is the dependency set of user $n_{i}$, i.e., the users in the set $L_{n_{i}}$ have the ability to interfere with $n_{i}$.

${ }^{1}$ This follows from the property of compactness, i.e., every cover has a finite subcover. 
Then, we have that $\lim _{l \rightarrow \infty} p_{n_{i+1}}\left(\hat{\epsilon}_{l}\right)=0$, where $i=0, \ldots, t-1$ and

$$
\lim _{l \rightarrow \infty} p_{m}\left(\hat{\epsilon}_{l}\right)=0 .
$$

Equation (15) holds for all users $m \in \mathcal{K}$. From our assumptions, we know that $\left\|p\left(\hat{\epsilon}_{l}\right)\right\|_{\infty}=1$. Therefore, there must be an index $k_{1} \in \mathcal{K}$ and an infinite sequence $\left\{l_{r}\right\}_{r \in \mathbb{N}}$ of natural numbers such that $p_{k_{1}}\left(\hat{\epsilon}_{l_{r}}\right)=1$ for all $r \in \mathbb{N}$. This, however, contradicts (15). Therefore (13) must be true.

Since (13) is true, there must exist a null-sequence $\left\{\tilde{\epsilon}_{l}\right\}_{l \in \mathbb{N}}$ (a null-sequence is a sequence that converges to 0 ) and a power vector $\boldsymbol{p}^{*}>\mathbf{0}$ such that $\lim _{l \rightarrow \infty} p_{k}\left(\tilde{\epsilon}_{l}\right)=p_{k}^{*}, k \in \mathcal{K}$. This follows from the compactness [23] of the following set: $\{\boldsymbol{p} \in$ $\left.\mathbb{R}_{+}^{K} \mid p_{k} \geq c_{1}, \forall k \in \mathcal{K},\|\boldsymbol{p}\|_{\infty}=1\right\}$. The fact that $p_{k}^{*} \geq c_{1}$ follows from (13).

Furthermore, for all $l \in \mathbb{N}$ and for all users $k \in \mathcal{K}$, we have that $\gamma_{k} \mathcal{I}_{k}\left(\boldsymbol{p}\left(\tilde{\epsilon}_{l}\right)\right) \leq\left(C(\boldsymbol{\gamma}, \mathcal{I})+\tilde{\epsilon}_{l}\right) p_{k}\left(\tilde{\epsilon}_{l}\right)$. From the continuity of the interference function $\mathcal{I}_{k}$, for all $k \in \mathcal{K}$, we have that

$$
\begin{aligned}
\gamma_{k} \mathcal{I}_{k}\left(\boldsymbol{p}^{*}\right) & =\lim _{l \rightarrow \infty} \gamma_{k} \mathcal{I}_{k}\left(\boldsymbol{p}\left(\tilde{\epsilon}_{l}\right)\right) \\
& \leq \lim _{l \rightarrow \infty}\left[\left(C(\boldsymbol{\gamma}, \mathcal{I})+\tilde{\epsilon}_{l}\right) p_{k}\left(\tilde{\epsilon}_{l}\right)\right] \\
& =C(\boldsymbol{\gamma}, \mathcal{I}) p_{k}^{*} .
\end{aligned}
$$

Therefore, we obtain $\gamma_{k} \mathcal{I}_{k}\left(\boldsymbol{p}^{*}\right) \leq C(\boldsymbol{\gamma}, \mathcal{I}) p_{k}^{*}$, for all $k \in \mathcal{K}$.

We have to show that (12) is satisfied. For the sake of obtaining a contradiction, let us assume that (12) is not satisfied. Then, there exists a user $\tilde{k} \in \mathcal{K}$ such that the following expression holds:

$$
\gamma_{\tilde{k}} \mathcal{I}_{\tilde{k}}\left(\boldsymbol{p}^{*}\right)<C(\boldsymbol{\gamma}, \mathcal{I}) p_{\tilde{k}}^{*}
$$

We now define a new power vector $\boldsymbol{p}^{(1)}$ such that $p_{k}^{(1)}=p_{k}^{*}$ for $k \neq \tilde{k}$. Furthermore, we choose $p_{\tilde{k}}^{(1)}<p_{\tilde{k}}^{*}$ such that we always have $\gamma_{\tilde{k}} \mathcal{I}_{\tilde{k}}\left(\boldsymbol{p}^{(1)}\right)<C(\boldsymbol{\gamma}, \mathcal{I}) p_{\tilde{k}}^{(1)}$.

Let $\mathcal{I}_{k_{1}}$ be any interference function corresponding to a user $k_{1}$ such that $[G]_{k_{1} \tilde{k}}>0$, i.e., $k_{1} \in L_{\tilde{k}}$. We denote the set of all users $k_{1}$ with $[\boldsymbol{G}]_{k_{1} \tilde{k}}>0$ as $\mathcal{M}_{1}$, i.e.,

$$
\mathcal{M}_{1}=\left\{k_{1} \mid[\boldsymbol{G}]_{k_{1} \tilde{k}}>0, k_{1}, \tilde{k} \in \mathcal{K}\right\} .
$$

The user $\tilde{k}$ is in $L_{k_{1}}$ (the dependency set of user $k_{1}$ ).

From the strict monotonicity of the interference function $\mathcal{I}_{k_{1}}$ and from $p_{\tilde{k}}^{(1)}<p_{\tilde{k}}^{*}$, we have that

$$
\begin{aligned}
\gamma_{k_{1}} \mathcal{I}_{k_{1}}\left(\boldsymbol{p}^{(1)}\right) & <\gamma_{k_{1}} \mathcal{I}_{k_{1}}\left(\boldsymbol{p}^{*}\right) \\
& \leq C(\boldsymbol{\gamma}, \mathcal{I}) p_{k_{1}}^{*} \\
& =C(\boldsymbol{\gamma}, \mathcal{I}) p_{k_{1}}^{(1)} .
\end{aligned}
$$

Therefore

$$
\gamma_{k_{1}} \mathcal{I}_{k_{1}}\left(\boldsymbol{p}^{(1)}\right)<C(\boldsymbol{\gamma}, \mathcal{I}) p_{k_{1}}^{(1)}
$$

Now, we can choose a new power vector $\boldsymbol{p}^{(2)}$ such that $p_{k}^{(2)}=$ $p_{k}^{(1)}$ for $k \in\{1, \ldots, K\} \backslash \mathcal{M}_{1}$. For $k_{1} \in \mathcal{M}_{1}$, we shall choose $p_{k_{1}}^{(2)}<p_{k_{1}}^{(1)}$ large enough, such that for the power vector $\boldsymbol{p}^{(2)}$ satisfies (17) for all $k_{1} \in \mathcal{M}_{1}$.
Now, based on similar arguments, we can construct another set $\mathcal{M}_{2}$, where

$$
\mathcal{M}_{2}=\left\{k_{2} \in \mathcal{K} \mid \exists k_{1} \in \mathcal{M}_{1} \text {, s.t. }[\boldsymbol{G}]_{k_{2} k_{1}}>0\right\} .
$$

From our precondition that matrix $G$ is irreducible, we can repeat this process finitely many times till $\mathcal{M}_{1} \cup \mathcal{M}_{2} \cup \ldots \cup \mathcal{M}_{n}$, where

$$
\mathcal{M}_{n}=\left\{k_{n} \in \mathcal{K} \mid \exists k_{n-1} \in \mathcal{M}_{n-1} \text {, s.t. }[\boldsymbol{G}]_{k_{n} k_{n-1}}>0\right\} .
$$

Hence, we obtain power vector $\boldsymbol{p}^{(m)}$ such that

$$
\gamma_{k} \mathcal{I}_{k}\left(p^{(m)}\right)<C(\boldsymbol{\gamma}, \mathcal{I}) p_{k}^{(m)} \quad \forall k \in \mathcal{K} .
$$

Naturally, we have that $\boldsymbol{p}^{(m)}>\mathbf{0}$. Furthermore, we have that

$$
\begin{aligned}
C(\boldsymbol{\gamma}, \mathcal{I}) & =\inf _{\boldsymbol{p}>\mathbf{0}} \max _{1 \leq k \leq K} \frac{\gamma_{k} \mathcal{I}_{k}(\boldsymbol{p})}{p_{k}} \\
& \leq \max _{1 \leq k \leq K} \frac{\gamma_{k} \mathcal{I}_{k}\left(\boldsymbol{p}^{(m)}\right)}{p_{k}^{(m)}} \\
& <C(\boldsymbol{\gamma}, \mathcal{I}) \quad \text { (inequality follows from (18)). }
\end{aligned}
$$

We have our desired contradiction. Hence, there does not exist an index $\tilde{k}$ such that (16) is valid. Therefore, for all users $k \in \mathcal{K}$, we have that the expression $\gamma_{k} \mathcal{I}_{k}\left(\boldsymbol{p}^{*}\right)=C(\boldsymbol{\gamma}, \mathcal{I}) p_{k}^{*}$ must hold. This proves our desired result.

Our second main result states that irreducibility of the matrix $\boldsymbol{G}$ is necessary and sufficient for Pareto optimality. This is a very strong result and carries over to utility sets, which can be obtained as a suitable mapping of the $\mathcal{S}$ set using (4).

Theorem 2: Let $\mathcal{I}_{1}, \ldots, \mathcal{I}_{K}$ be strictly positive and strictly monotonic interference functions on their respective dependency set. Then, all boundary points are Pareto-optimal if and only if the global coupling matrix $G$ is irreducible.

Proof: " $\Longleftarrow$ ": Let $\boldsymbol{\gamma}^{(1)}$ be any boundary point of a QoS set, such that $C\left(\boldsymbol{\gamma}^{(1)}, \mathcal{I}\right)=1$ is not Pareto-optimal. Then, there must exist a $\boldsymbol{\gamma}^{(2)} \geq \boldsymbol{\gamma}^{(1)}, \boldsymbol{\gamma}^{(2)} \neq \boldsymbol{\gamma}^{(1)}$ with $C\left(\boldsymbol{\gamma}^{(2)}, \mathcal{I}\right)=$ 1. Let $\boldsymbol{p}^{*}=\boldsymbol{p}^{*}\left(\boldsymbol{\gamma}^{(2)}\right)$ be the corresponding vector from Theorem 1. Therefore, we have that $\boldsymbol{p}^{*}>0$ and $\gamma_{k}^{(2)} \mathcal{I}_{k}\left(\boldsymbol{p}^{*}\right)=$ $C\left(\boldsymbol{\gamma}^{(2)}, \mathcal{I}\right) p_{k}^{*}, 1 \leq k \leq K$. Thus, we have that $\gamma_{k}^{(1)} \mathcal{I}_{k}\left(\boldsymbol{p}^{*}\right) \leq$ $p_{k}^{*}$, and from $\boldsymbol{\gamma}^{(2)} \geq \boldsymbol{\gamma}^{(1)}, \boldsymbol{\gamma}^{(2)} \neq \boldsymbol{\gamma}^{(1)}$ there must exist at least a singular index $\tilde{k}$ with $\gamma_{\tilde{k}}^{(1)} \mathcal{I}_{\tilde{k}}\left(\boldsymbol{p}^{*}\right)<p_{\tilde{k}}^{*}$. Similarly as in the proof of Theorem 1, we can find a vector $\boldsymbol{p}^{m}>\mathbf{0}$, such that $\gamma_{k}^{(1)} \mathcal{I}_{k}\left(\boldsymbol{p}^{(m)}\right)<p_{k}^{(m)}, 1 \leq k \leq K$. However then, similarly as in the proof of Theorem $1, C\left(\boldsymbol{\gamma}^{(1)}, \mathcal{I}\right)<1$. Then, $\boldsymbol{\gamma}^{(1)}$ is not a boundary point and contradicts our assumptions. Therefore, every boundary point is Pareto-optimal.

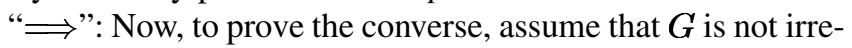
ducible, i.e., $\boldsymbol{G}$ is reducible. This implies that there are boundary points, which are not Pareto-optimal, i.e., weak Pareto-optimal. Since $\boldsymbol{G}$ is reducible, through a simultaneous permutation of the rows and columns of $\boldsymbol{G}$, we can achieve the following form:

$$
\boldsymbol{G}=\left(\begin{array}{cc}
\boldsymbol{G}^{(1)} & \mathbf{0} \\
\boldsymbol{G}^{(1,2)} & \boldsymbol{G}^{(2)}
\end{array}\right)
$$

where the matrix $\boldsymbol{G}^{(1)}\left(\boldsymbol{G}^{(1)}\right.$ is a $k_{1} \times k_{1}$ matrix $)$ is irreducible. 
We assume that $\mathcal{I}_{1}, \ldots, \mathcal{I}_{K}$ are prepared in the corresponding required form. Therefore, $\mathcal{I}_{k}, 1 \leq k \leq k_{1}$, is dependent only on $p_{l}, 1 \leq l \leq k_{1}$. We now analyze the following equations:

and

$$
C(\boldsymbol{\gamma}, \mathcal{I})=\inf _{\boldsymbol{p}>0} \max _{1 \leq k \leq K} \frac{\gamma_{k} \mathcal{I}_{k}(\boldsymbol{p})}{p_{k}}
$$

$$
C\left(\boldsymbol{\gamma}^{(1)}, \mathcal{I}^{(1)}\right)=\inf _{\boldsymbol{p}^{(1)} \in \mathbb{R}_{+, 0}^{K, k_{1}}, \boldsymbol{p}^{(1)}>\mathbf{0}} \max _{1 \leq k \leq k_{1}} \frac{\gamma_{k} \mathcal{I}_{k}(\boldsymbol{p})}{p_{k}}
$$

with power vector $\boldsymbol{p}^{(1)}=\left[p_{1}, \ldots, p_{k_{1}}\right]^{T}$, interference function $\mathcal{I}^{(1)}=\left[\mathcal{I}_{1}, \ldots, \mathcal{I}_{k_{1}}\right]^{T}$, and matrix $\gamma=\operatorname{diag}\left(\gamma_{1}, \ldots, \gamma_{K}\right)$.

The interference-balancing function $C\left(\boldsymbol{\gamma}^{(2)}, \mathcal{I}^{(2)}\right)$ can be written as

$$
C\left(\boldsymbol{\gamma}^{(2)}, \mathcal{I}^{(2)}\right)=\inf _{\boldsymbol{p} \in \mathbb{R}_{++}^{K}} \max _{k_{1}<k \leq K} \frac{\gamma_{k} \mathcal{I}_{k}(\boldsymbol{p})}{p_{k}} .
$$

From Lemma 3, we know that $C\left(\boldsymbol{\gamma}^{(1)}, \mathcal{I}^{(1)}\right)>0$ and that $C\left(\boldsymbol{\gamma}^{(2)}, \mathcal{I}^{(2)}\right)>0$. From Lemma 4 , we know that $C(\boldsymbol{\gamma}, \mathcal{I})=$ $\max \left(C\left(\boldsymbol{\gamma}^{(1)}, \mathcal{I}^{(1)}\right), C\left(\boldsymbol{\gamma}^{(2)}, \mathcal{I}^{(2)}\right)\right)$.

We now choose a vector $\gamma$ such that

$$
C(\boldsymbol{\gamma}, \mathcal{I})=C\left(\boldsymbol{\gamma}^{(1)}, \mathcal{I}^{(1)}\right)
$$

Therefore, we have that $0<C\left(\boldsymbol{\gamma}^{(2)}, \mathcal{I}^{(2)}\right) \leq 1$. For $0<\lambda<1$, we choose a vector $\gamma(\lambda)$ with

$$
\gamma_{k}(\lambda)=\gamma_{k}, \quad \text { for } 1 \leq k \leq k_{1}
$$

and

$$
\gamma_{k}(\lambda)=\lambda \gamma_{k}, \quad \text { for } k_{1}<k \leq K+1 .
$$

Then, we have that

$$
\begin{aligned}
C(\boldsymbol{\gamma}(\lambda), \mathcal{I}) & \geq C\left(\boldsymbol{\gamma}^{(1)}(\lambda), \mathcal{I}^{(1)}\right) \\
& =C\left(\boldsymbol{\gamma}^{(1)}, \mathcal{I}^{(1)}\right) \\
& =1 \\
C(\boldsymbol{\gamma}(\lambda), \mathcal{I}) & =\inf _{\boldsymbol{p}>\mathbf{0}} \max _{1 \leq k \leq K}\left(\gamma_{k}(\lambda) \mathcal{I}_{k}(\boldsymbol{p})\right) / p_{k} \\
& \leq \inf _{\boldsymbol{p}>\mathbf{0}} \max _{1 \leq k \leq K}\left(\gamma_{k} \mathcal{I}_{k}(\boldsymbol{p})\right) / p_{k} \\
& =1 .
\end{aligned}
$$

Then, from (20) and (21), we can conclude that

$$
C(\gamma(\lambda), \mathcal{I})=1 .
$$

Therefore, we obtain that the SIR vector $\boldsymbol{\gamma}(\lambda)$ is a boundary point. Naturally $\boldsymbol{\gamma}(\lambda) \leq \boldsymbol{\gamma}$ and $\boldsymbol{\gamma}(\lambda) \neq \boldsymbol{\gamma}$ for $\lambda \neq 1$. Hence, we are led to the conclusion that the SIR vector $\gamma(\lambda)$ is only a weak Pareto-optimal boundary point.

We present an example for a four-user case, where we identify the set of Pareto-optimal and the set of weak Pareto-optimal points.
Example 2: Consider the reducible coupling matrix $V$ given by

$$
\boldsymbol{V}=\left(\begin{array}{cccc}
0 & \rho_{1} & 0 & 0 \\
\rho_{1} & 0 & 0 & 0 \\
1 & 1 & 0 & \rho_{2} \\
1 & 1 & \rho_{2} & 0
\end{array}\right)
$$

and the corresponding global dependency matrix $\boldsymbol{G}$ given by

$$
\boldsymbol{G}=\left(\begin{array}{llll}
0 & 1 & 0 & 0 \\
1 & 0 & 0 & 0 \\
1 & 1 & 0 & 1 \\
1 & 1 & 1 & 0
\end{array}\right)
$$

This is an example of a system of four users with no self-interference. User 1 and user 2 see no interference except from each other, while user 3 and user 4 see interference from all the other three users, respectively.

We shall briefly utilize the matrix-based interference model. For linear interference models $\mathcal{I}_{k}(\boldsymbol{p})=[\boldsymbol{V} \boldsymbol{p}]_{k}$, for $k \in\{1,2,3,4\}$, the resulting SIR is given by

$$
\mathrm{SIR}_{k}=\frac{p_{k}}{[\boldsymbol{V} \boldsymbol{p}]_{k}} \quad \forall k \in\{1,2,3,4\} .
$$

Let $\gamma_{1}, \gamma_{2}, \gamma_{3}$, and $\gamma_{4}$ be the SIR values of users $1-4$, respectively, and let $\boldsymbol{\Gamma}=\operatorname{diag}\left(\gamma_{1}, \ldots, \gamma_{4}\right)$. For the boundary points, we have that $C(\boldsymbol{\Gamma}, \mathcal{I})=1$, where

$$
C(\boldsymbol{\Gamma}, \mathcal{I})=\inf _{\boldsymbol{p}>\mathbf{0}:\|\boldsymbol{p}\|_{1}=1} \max _{k \in\{1,2,3,4\}}\left(\frac{[\boldsymbol{\Gamma} \boldsymbol{V}]_{k}}{p_{k}}\right)
$$

where

$$
\boldsymbol{V}=\left(\begin{array}{cc}
\boldsymbol{V}^{(1)} & \mathbf{0} \\
\boldsymbol{V}^{(1,2)} & \boldsymbol{V}^{(2)}
\end{array}\right)
$$

Furthermore

$$
\boldsymbol{\Gamma} \boldsymbol{V}=\left(\begin{array}{cc}
\boldsymbol{\Gamma}^{(1)} \boldsymbol{V}^{(1)} & \mathbf{0} \\
\boldsymbol{\Gamma}^{(1,2)} \boldsymbol{V}^{(1,2)} & \boldsymbol{\Gamma}^{(2)} \boldsymbol{V}^{(2)}
\end{array}\right)
$$

We know that

$$
C(\boldsymbol{\Gamma}, \mathcal{I})=\max \left(C\left(\boldsymbol{\Gamma}^{(1)}, \mathcal{I}^{(1)}\right), C\left(\boldsymbol{\Gamma}^{(2)}, \mathcal{I}^{(2)}\right)\right) .
$$

Therefore, we have that $C\left(\boldsymbol{\Gamma}^{(1)}, \mathcal{I}^{(1)}\right)=\rho_{1}^{2} \gamma_{1} \gamma_{2}$ and $C\left(\boldsymbol{\Gamma}^{(2)}, \mathcal{I}^{(2)}\right)=\rho_{2}^{2} \gamma_{3} \gamma_{4}$ (since $C(\boldsymbol{\Gamma}, \mathcal{I})=1$. The Pareto-optimal boundary points are given by the set $\mathbf{P O}$, where $\mathbf{P O}=\left\{\left(\gamma_{1}, \gamma_{2}, \gamma_{3}, \gamma_{4}\right) \in \mathbb{R}_{++}^{4} \mid \gamma_{1} \gamma_{2}=\frac{1}{\rho_{1}^{2}}, \gamma_{3} \gamma_{4}=\frac{1}{\rho_{2}^{2}}\right\}$. The Pareto-optimal boundary points are displayed in Fig. 1. The weak Pareto-optimal boundary points are given by the set WPO, where WPO $=\mathbf{S}_{1} \cup \mathbf{S}_{2}$, $\mathbf{S}_{1}=\left\{\left(\gamma_{1}, \gamma_{2}, \gamma_{3}, \gamma_{4}\right) \in \mathbb{R}_{+}^{4} \mid \gamma_{1} \gamma_{2}=\frac{1}{\rho_{1}^{2}}, \gamma_{3} \gamma_{4}<\frac{1}{\rho_{2}^{2}}\right\}$ and $\mathbf{S}_{2}=\left\{\left(\gamma_{1}, \gamma_{2}, \gamma_{3}, \gamma_{4}\right) \mid \gamma_{1} \gamma_{2}<\frac{1}{\rho_{1}^{2}}, \gamma_{3} \gamma_{4}=\frac{1}{\rho_{2}^{2}}\right\}$. The sets $\mathbf{S}_{1}$ and $\mathbf{S}_{2}$ are depicted in Figs. 2 and 3, respectively, since we have a four-dimensional region for four users. We have depicted each set as two separate figures, as it is impossible to draw a four-dimensional set.

For all interference functions with $K=4$ and with the global dependency matrix $\boldsymbol{G}$ as defined by (22), we have for 

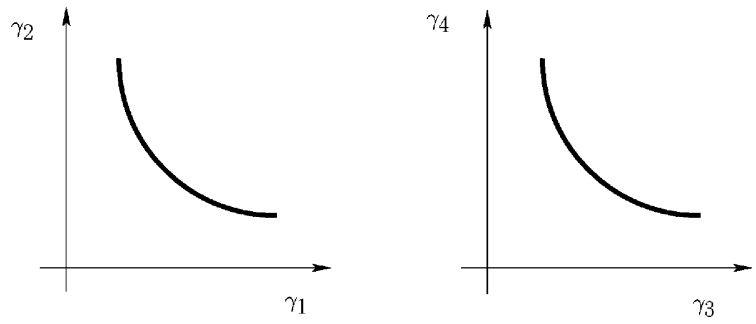

Fig. 1. Pareto-optimal boundary points corresponding to coupling matrix $\boldsymbol{V}$.
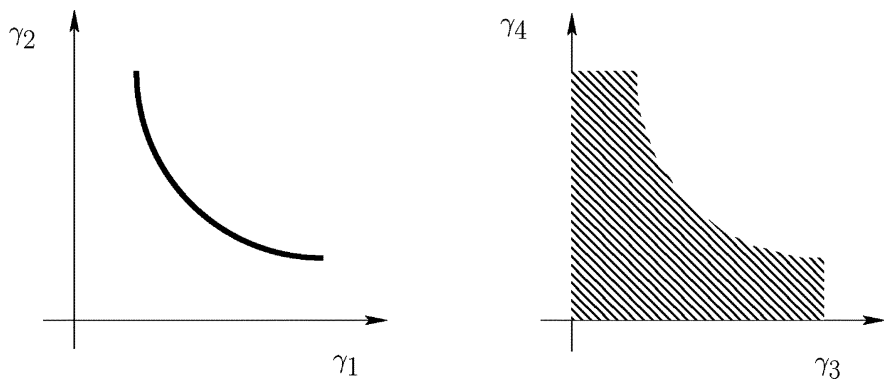

Fig. 2. Weak Pareto-optimal boundary points corresponding to coupling matrix $\boldsymbol{V}$ and set $\mathbf{S}_{1}$. The shaded region corresponds to the inequality and the weak Pareto-optimal points.
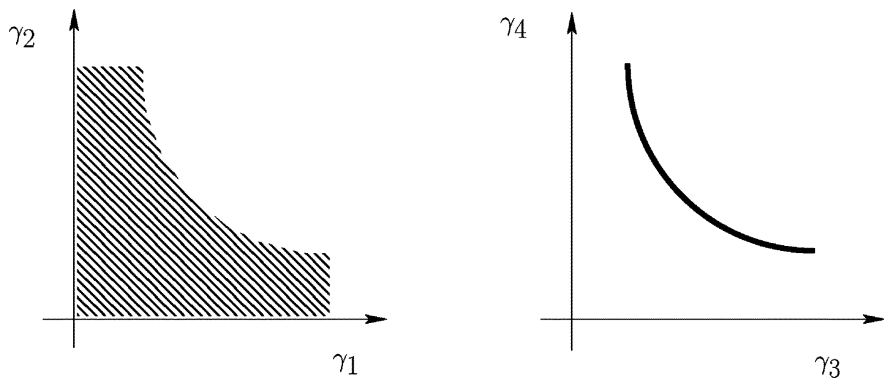

Fig. 3. Weak Pareto-optimal boundary points corresponding to coupling matrix $\boldsymbol{V}$ and set $\mathbf{S}_{2}$. The shaded region corresponds to the inequality and the weak Pareto optimal points.

the case of user $1, \mathcal{I}_{1}\left(\left[p_{1}, p_{2}, p_{3}, p_{4}\right]\right)=\mathcal{I}_{1}\left(p_{2}\right)=p_{2} \mathcal{I}_{1}(1)$ and $\mathcal{I}_{1}\left(\lambda p_{2}\right)=\lambda \mathcal{I}_{1}\left(p_{2}\right)$, where $\lambda>0$ is a scalar such that $\lambda=\frac{1}{p_{2}}$, from $A 2$. Then, we have that interference function $\mathcal{I}_{1}(1) \stackrel{p_{2}}{=} \mathcal{I}_{1}\left(p_{2}\right) / p_{2}$ and $\mathcal{I}_{1}\left(p_{2}\right)=p_{2} \mathcal{I}_{1}(1)$. A similar analysis can be carried out for user 2 , where interference function $\mathcal{I}_{2}\left(p_{1}\right)=p_{1} \mathcal{I}_{2}(1)$. Analyzing the interference functions for users 3 and 4, we have that $\inf _{\boldsymbol{p}>0} \max _{k \in\{3,4\}} \gamma_{k} \mathcal{I}_{k}(\boldsymbol{p}) / p_{k}=$ $\inf _{p_{3}, p_{4}>0, p_{1}=p_{2}=0} \max _{k \in\{3,4\}} \gamma_{k} \mathcal{I}_{k}(\boldsymbol{p}) p_{k}$, whose value is $\gamma_{3} \gamma_{4} \mathcal{I}_{3}\left((0,0,0,1)^{T}\right) \mathcal{I}_{4}\left((0,0,1,0)^{T}\right)$. The resulting SIR vectors $\left(\gamma_{1}, \gamma_{2}, \gamma_{3}, \gamma_{4}\right)$ are exactly the Pareto-optimal boundary points.

We shall now state a generalization of the property of the global dependency matrix, which was encountered in Example 2. Let it be possible through an appropriate simultaneous permutation of rows and columns to obtain the global dependency matrix $G$ in the normal form

$$
\boldsymbol{G}=\left(\begin{array}{cc}
\boldsymbol{A}_{1} & 0 \\
\mathbf{1} & \boldsymbol{A}_{1}
\end{array}\right)
$$

where the matrix $\boldsymbol{A}_{1}$, depicting only one user interfering with any other user, is

$$
\boldsymbol{A}_{1}=\left(\begin{array}{llll}
0 & 1 & 0 & 0 \\
0 & 0 & 1 & 0 \\
0 & 0 & 0 & 1 \\
1 & 0 & 0 & 0
\end{array}\right)
$$

and the matrix 1 is

$$
\mathbf{1}=\left(\begin{array}{llll}
1 & 1 & 1 & 1 \\
1 & 1 & 1 & 1 \\
1 & 1 & 1 & 1 \\
1 & 1 & 1 & 1
\end{array}\right)
$$

Then, we can calculate the Pareto-optimal boundary points as shown in Example 2.

\section{ANALYSIS OF PARETO-Optimal Boundary of Utility SETS With INDIVIDUAL POWER CONSTRAINTS}

In this section, we shall analyze the properties of boundary points of utility sets with individual power constraints.

Remark 6: Here, we have that $\mathcal{P}:=\{\boldsymbol{p} \mid \boldsymbol{p} \leq \hat{\boldsymbol{p}}\}$, where $\hat{\boldsymbol{p}}=$ $\left[\hat{p}_{1}, \ldots, \hat{p}_{K}\right]$ are the individual power constraints. In this case, we have the following power vector $\boldsymbol{p} \in \mathcal{P}$, here $\mathcal{P}=\mathbb{R}_{+}^{K+1}$, i.e., we have that $G$ is a $K \times(K+1)$-dimensional matrix.

We shall utilize certain proof techniques developed in Section III for obtaining the results in this section. We begin by presenting the structure of the QoS region for individual power constraints.

\section{A. Structure of the QoS Region for Individual Power Constraints}

Consider the SINR feasible region for users $\mathcal{K}:=\{1, \ldots, K\}$ with individual power constraints $\boldsymbol{p} \leq \boldsymbol{p}^{\mathrm{max}}$, which is defined as the sublevel set

$$
\mathcal{S}\left(\mathcal{I}, \boldsymbol{p}^{\max }\right)=\left\{\boldsymbol{\gamma} \in \mathbb{R}_{+}^{K}: C\left(\boldsymbol{\gamma}, \mathcal{I}, \boldsymbol{p}^{\max }\right) \leq 1\right\}
$$

where $\gamma$ is a vector of SINR values, whose feasibility is determined by the min-max optimum (see, e.g., [20] and [21])

$$
C\left(\boldsymbol{\gamma}, \mathcal{I}, \boldsymbol{p}^{\max }\right)=\inf _{\boldsymbol{0} \leq \boldsymbol{p} \leq \boldsymbol{p}^{\max }} \max _{k \in \mathcal{K}} \frac{\gamma_{k} \mathcal{I}_{k}(\underline{\boldsymbol{p}})}{p_{k}} .
$$

The structure of the SINR set $\mathcal{S}\left(\mathcal{I}, p^{\max }\right)$ depends on the properties of $C\left(\boldsymbol{\gamma}, \mathcal{I}, p^{\max }\right)$, which in turn depends on the properties of the underlying interference functions $\mathcal{I}_{1}, \ldots, \mathcal{I}_{K}$ as well as on the chosen power constraints $\boldsymbol{p}^{\max }$. The corresponding QoS values $\boldsymbol{u} \in \mathbf{U}$ are feasible if and only if $C\left(\boldsymbol{\gamma}(\boldsymbol{u}), \mathcal{I}, \boldsymbol{p}^{\max }\right) \leq 1$. The QoS feasible set is the sublevel set

$$
\mathbf{U}=\left\{\boldsymbol{u} \in \mathbb{R}_{+}^{K} \mid C\left(\boldsymbol{\gamma}(\boldsymbol{u}), \mathcal{I}, \boldsymbol{p}^{\max }\right) \leq 1\right\} .
$$

We are now interested in the boundary of $\mathbf{U}$, which is characterized by $C\left(\boldsymbol{\gamma}(\boldsymbol{u}), \mathcal{I}, \boldsymbol{p}^{\max }\right)=1$. The boundary is denoted as $\partial \mathbf{U}$. Since $\mathcal{I}_{1}, \ldots, \mathcal{I}_{K}$ are strictly monotonic in $p_{K+1}$, there exists a $\boldsymbol{\gamma}>\mathbf{0}$ such that $\boldsymbol{p}=\boldsymbol{p}(\boldsymbol{\gamma}), p_{k} \leq p_{k}^{\max }$, for $K \geq 2$ with $\gamma_{k} \mathcal{I}_{k}(\boldsymbol{p})=C\left(\boldsymbol{\gamma}, \mathcal{I}, \boldsymbol{p}^{\max }\right) p_{k}, 1 \leq k \leq K$. We now introduce 
the restricted global dependency matrix, which shall be used to obtain certain results in the following section.

Definition 7: Restricted global dependency matrix. $G$, the global dependency matrix, is a $K \times(K+1)$ matrix, and the dependency on noise is clear for every $\mathcal{I}_{1}, \ldots, \mathcal{I}_{K}$. Then

$$
\boldsymbol{G}=\left(\begin{array}{l|c}
\boldsymbol{G}_{\mathrm{Res}} & \vdots \\
& 1
\end{array}\right)
$$

where $G_{\text {Res }}$ is the restricted global dependency matrix of dimension $K \times K$ and $[1, \ldots, 1]^{T}$ is representing the dependency on noise.

Remark 7: $\boldsymbol{G}_{\text {Res }}$ measures the "crosstalk" in the system, i.e., the dependency between the users due to direct interference (as opposed to indirect dependency due to power constraints).

\section{B. Pareto-Optimal Boundary of Utility Sets With Individual Power Constraints}

As can be seen from Section IV-A, with individual power constraints, the structure of the SIR region, and in turn of certain utility regions using (4), depends not only on the interference coupling, but also on the noise. The following result describes the conditions for the boundary points of utility sets with individual power constraints to be Pareto-optimal, i.e., it specifies the conditions on the interference coupling of multiuser systems with individual power constraints such that any operating point chosen by a resource allocation strategy on the boundary of the utility set is efficient.

Theorem 3: Let interference functions $\mathcal{I}_{1}, \ldots, \mathcal{I}_{K}$ be strictly monotonic on their respective dependency sets. Then, each boundary point is Pareto-optimal if and only if $\boldsymbol{G}_{\text {Res }}$ is irreducible.

Proof: "£": Let $\boldsymbol{G}_{\text {Res }}$ be irreducible. Let SINR vector $\boldsymbol{\gamma}^{(1)}$ be a boundary point, which is not Pareto-optimal. Then, there exists a SINR vector $\boldsymbol{\gamma}^{(2)}$ such that $1=$ $C\left(\boldsymbol{\gamma}^{(1)}, \mathcal{I}, \boldsymbol{p}^{\max }\right)=C\left(\boldsymbol{\gamma}^{(2)}, \mathcal{I}, \boldsymbol{p}^{\max }\right)$, where $C\left(\boldsymbol{\gamma}, \mathcal{I}, \boldsymbol{p}^{\max }\right)=$

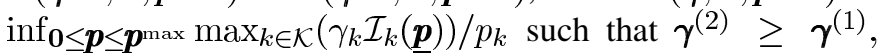
$\boldsymbol{\gamma}^{(2)} \neq \boldsymbol{\gamma}^{(1)}$

Let there be a power vector $\boldsymbol{p}^{(2)}$ such that $p_{k}^{(2)} \leq p_{k}^{\max }$, $1 \leq k \leq K$. The solution is $\gamma_{k}^{(2)} \mathcal{I}_{k}\left(\boldsymbol{p}^{(2)}\right)=\boldsymbol{p}^{(2)}, 1 \leq k \leq$ $K$. There exists exactly one such vector. This follows from [6, p. 1343, Lemma 1] and Lemma 2. Therefore, we have that $\gamma_{k}^{(1)} \mathcal{I}_{k}\left(\boldsymbol{p}^{(2)}\right) \leq p_{k}^{(2)}$ and that there exists at least one $\tilde{k}$ such that $\gamma_{\tilde{k}}^{(1)} \mathcal{I}_{\tilde{k}}\left(\boldsymbol{p}^{(2)}\right)<p_{\tilde{k}}^{(2)}$. Then, one can carry out the same process as in the proof of Theorem 1 with $\boldsymbol{G}_{\text {Res }}$ being our irreducible matrix. We can construct a vector $\boldsymbol{p}^{(m)}$ with $\boldsymbol{p}^{(m)} \leq \boldsymbol{p}^{(2)}$ such that $\gamma_{k}^{(1)} \mathcal{I}_{k}\left(\boldsymbol{p}^{(m)}\right)<p_{k}^{(m)}, 1 \leq k \leq K$. The vector $\boldsymbol{p}^{(m)}$ fulfills the power constraints, and we have $C\left(\boldsymbol{\gamma}^{(1)}, \mathcal{I}, \boldsymbol{p}^{\max }\right)<1$, which gives us the desired contradiction. Hence, if $\boldsymbol{G}_{\text {Res }}$ is irreducible, then every boundary point is Pareto-optimal.

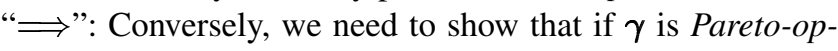
timal, then the restricted global dependency matrix is irreducible. Suppose that $\boldsymbol{G}_{\text {Res }}$ is reducible and $\boldsymbol{G}_{\text {Res }}$ is in Frobenius normal form [24], with irreducible matrices $\boldsymbol{G}_{\text {Res }, 1}, \ldots, \boldsymbol{G}_{\text {Res }, K}$ along the main diagonal. Here, $\boldsymbol{G}_{\text {Res }, 1}, \ldots, \boldsymbol{G}_{\text {Res }, K}$ have dimensions $k_{1} \times k_{1}, \ldots, k_{N} \times k_{N}$, respectively, and $\sum_{l=1}^{N} k_{l}=K$. Such a canonical form

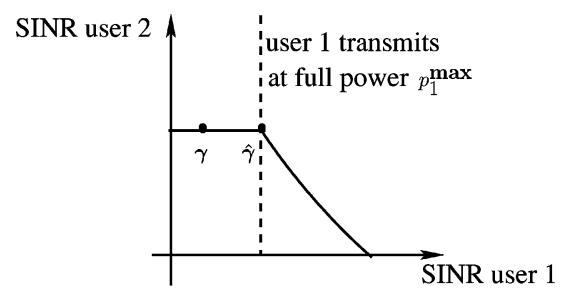

Fig. 4. SINR feasible region for the two-user MAC channel described in Example 3.

can always be achieved by an appropriate permutation simultaneously applied to row and column indices. Suppose that the first (isolated) block has dimensions $k_{1} \times k_{1}$. That is, the first $k_{1}$ interference functions only depend on the first $k_{1}$ components of $\boldsymbol{p}$ (and also strictly monotonic with respect to the noise component). There exists a vector $\boldsymbol{p}^{*}$ with $\boldsymbol{p}^{*}=\gamma_{k} \mathcal{I}_{k}\left(\boldsymbol{p}^{*}\right)$. Consider the vector $\boldsymbol{p}^{*}=\left(\boldsymbol{p}_{0}^{*} p_{n}^{*}\right)$, where $\boldsymbol{p}_{0}^{*}=\left[p_{1}^{*}, \ldots, p_{k_{1}}^{*}\right]^{T}$. For all $1 \leq k \leq k_{1}$, we have $p_{k}^{*}=\gamma_{k} \mathcal{I}_{k}\left(\underline{\boldsymbol{p}}_{0}^{*}\right)$. Introducing $\overline{\boldsymbol{p}}^{(1)}=\left[p_{1}^{\max }, \ldots, p_{k_{1}}^{\max }\right]^{T}$, $\mathcal{I}^{(1)}=\left(\mathcal{I}_{1}, \ldots, \mathcal{I}_{k_{1}}\right), \boldsymbol{\gamma}^{(1)}=\left(\gamma_{1}, \ldots, \gamma_{k_{1}}\right)$, we have that $C_{1}\left(\boldsymbol{\gamma}^{(1)}, \boldsymbol{I}^{(1)}, \overline{\boldsymbol{p}}^{(1)}\right)=\inf _{\mathbf{0}_{k_{1}}<\boldsymbol{p} \leq \overline{\boldsymbol{p}}^{(1)}} \frac{\left.\gamma_{k} \mathcal{I}_{k} \underline{\boldsymbol{p}}_{0}\right)}{p_{k}}=1$. We can now focus on $\gamma_{k}=\lambda \gamma_{k}, k_{1}+1 \leq k \leq K, \lambda<1$. With $\gamma_{k}(\lambda)=\gamma_{k}, 1 \leq k \leq k_{1}$, we have

$$
\begin{aligned}
1 & \geq \inf _{\mathbf{0}<\boldsymbol{p}^{\prime} \boldsymbol{p}^{\max }} \max _{k \in \mathcal{K}} \frac{\gamma_{k} \mathcal{I}_{k}(\underline{\boldsymbol{p}})}{p_{k}} \\
& \geq \inf _{\mathbf{0}<\boldsymbol{p} \leq \boldsymbol{p}^{\max }} \max _{k \in\left\{1, \ldots, k_{1}\right\}} \frac{\gamma_{k} \mathcal{I}_{k}(\underline{\boldsymbol{p}})}{p_{k}} .
\end{aligned}
$$

The right-hand side of this inequality equals 1 . Hence, $C\left(\boldsymbol{\gamma}(\lambda), \mathcal{I}, \boldsymbol{p}^{\max }\right)=1$. Thus, $\boldsymbol{\gamma}(\lambda)$ is a boundary point. However, all components $\gamma_{k}(\lambda), k \geq k_{1}+1$ can be increased without affecting $\gamma_{k}(\lambda)=\gamma_{k}, 1 \leq k \leq k_{1}$. That is, $\gamma(\lambda)$ is not Pareto-optimal, which is our required contradiction.

The above result says that for all SIR vectors $\boldsymbol{\gamma}$, such that we can find a power vector $\boldsymbol{p} \leq \boldsymbol{p}^{\text {max }}$, we have that $\gamma_{k} \leq p_{k} / \mathcal{I}_{k}(\boldsymbol{p})$, $1 \leq k \leq K, \boldsymbol{p} \leq \boldsymbol{p}^{\max }$, and there exists an index $k_{0}$ such that $\gamma_{k_{0}}<\bar{p}_{k_{0}} / \mathcal{I}_{k_{0}}(\boldsymbol{p})$, for all $1 \leq k \leq K$. Then, we can find a power vector $0<\boldsymbol{p}^{*}<\boldsymbol{p}$ such that $\gamma_{k}<p_{k}^{*} / \mathcal{I}_{k}\left(\boldsymbol{p}^{*}\right)$, if and only if the matrix $\boldsymbol{G}_{\text {Res }}$ is irreducible. Furthermore, knowing the properties when the boundary points are Pareto-optimal aid us in designing appropriate algorithms for resource allocation and utility maximization.

Example 3: Consider a two-user Gaussian multiple access channel (MAC) with successive interference cancellation, normalized noise $\sigma_{n}^{2}=1$, and a given decoding order 1,2 . The SINR of the users are $\operatorname{SINR}_{1}(\boldsymbol{p})=\frac{p_{1}}{p_{2}+1}$ and $\operatorname{SINR}_{2}(\boldsymbol{p})=p_{2}$. Assuming power constraints $p_{1} \leq p_{1}^{\max }=1$ and $p_{2} \leq p_{2}^{\max }=1$, we obtain an SINR region as depicted in Fig. 4. Consider the boundary point $\gamma$ depicted in Fig. 4. This point $\gamma$ is achieved by $\boldsymbol{p}^{*}=\left[p_{1}^{\max } / 2, p_{2}^{\max } / 2\right]^{T}$. Hence, $\boldsymbol{p}^{*} \in \mathcal{P}\left(\boldsymbol{\gamma}, \boldsymbol{p}^{\max }\right)$. This vector achieves the SINR with component-wise minimum power. However, power vector $\boldsymbol{p}^{*}$ is not the only element of $\mathcal{P}\left(\boldsymbol{\gamma}, \boldsymbol{p}^{\max }\right)$. Due to interference cancellation, we can increase the power, hence the SINR of user 1, without reducing the SINR of user 2 . If both users transmit with maximum power $p^{\max }$, then the corner point $\hat{\gamma}$ is achieved. In 
addition, this power vector is contained in $\mathcal{P}\left(\boldsymbol{\gamma}, \boldsymbol{p}^{\max }\right)$ since $\hat{\gamma} \geq \gamma$, such that the SIR targets $\gamma$ are still fulfilled. For an arbitrary power vector $\boldsymbol{p} \in \mathcal{P}\left(\boldsymbol{\gamma}, \boldsymbol{p}^{\max }\right)$, consider the fixed-point iteration $p_{k}^{(n+1)}=\gamma_{k} \mathcal{I}_{k}\left(\underline{\boldsymbol{p}}^{(n)}\right), p_{k}^{(0)}=p_{k}$, for all $k \in \mathcal{K}$. The limit $\boldsymbol{p}^{*}=\lim _{n \rightarrow \infty} \boldsymbol{p}^{(n)}>0$ is special since it achieves the SIR vector $\gamma$ with component-wise minimum power.

\section{Analysis of the PARETO-Optimal BoUndary of UTILITy SETS With A TOTAL POWER CONSTRAINT}

In this section, we shall analyze the properties of boundary points of utility sets with a total power constraint.

Remark 8: Here, we have that $\mathcal{P}:=\{\boldsymbol{p} \mid \boldsymbol{p}=$ $\left.\left[p_{1}, \ldots, p_{K}\right], \sum_{k \in \mathcal{K}} p_{k} \leq P_{\text {total }}\right\}$, where $P_{\text {total }}$ is the total power constraint. In this case, we have the following power vector $\underline{p} \in \mathcal{P}$, here $\mathcal{P}=\mathbb{R}_{+}^{K+1}$, with a total power constraint on the vector $\boldsymbol{p} \in \mathbb{R}_{+}^{K}$ of $\sum_{k \in \mathcal{K}} p_{k} \leq P_{\text {total }}$.

Here, we consider interference functions from the Yates framework, which are strictly monotonic in the noise component. These results have direct impact on practical resource allocation strategies for utility sets of the specified type. We shall utilize certain proof techniques developed in Section III for obtaining the results in this section. We begin by presenting the structure of the QoS region for a total power constraint.

\section{A. Structure of the Qos Region With a Total Power Constraint}

The SINR region under a sum power constraint (total power constraint) is defined as

$$
\mathcal{S}\left(\mathcal{I}, P_{\text {total }}\right)=\left\{\boldsymbol{\gamma} \in \mathbb{R}_{+}^{K} \mid C\left(\boldsymbol{\gamma}, \mathcal{I}, P_{\text {total }}\right) \leq 1\right\}
$$

where

$$
C\left(\boldsymbol{\gamma}, \mathcal{I}, P_{\text {total }}\right)=\inf _{\boldsymbol{p}>\mathbf{0},\|\boldsymbol{p}\|_{1} \leq P_{\text {total }}} \max _{k \in \mathcal{K}} \frac{\gamma_{k} \mathcal{I}_{k}(\underline{\boldsymbol{p}})}{p_{k}} .
$$

We shall see in the next section (Section V) that the sum-power constrained region $\mathcal{S}\left(\mathcal{I}, P_{\text {total }}\right)$ is relatively easy to handle since the SINR tradeoffs are caused due to the sharing of a common power resource.

\section{B. Pareto-Optimal Boundary of Utility Sets With a Total Power Constraint}

Let us assume that the sum of all transmission powers is limited by $P_{\text {total }}$. The next result shows that for arbitrary interference functions, which are strictly monotonic with respect to noise, the boundary points of utility sets with a total power constraint and no self-interference are Pareto-optimal. The result states that for the case of multiuser systems with a total power constraint and no self-interference, all types of interference coupling result in all boundary points of the utility set are efficient.

Theorem 4: Let $\mathcal{I}_{1}, \ldots, \mathcal{I}_{K}$ be arbitrary interference functions. Then, for all $0<P_{\text {total }}<+\infty$, all boundary points of $\mathcal{S}\left(\mathcal{I}, P_{\text {total }}\right)$ are Pareto-optimal.

Proof: Consider the case of a system with a total power constraint such that $\sum_{k \in \mathcal{K}} p_{k} \leq P_{\text {total }}$ and $\boldsymbol{p} \geq \mathbf{0}$. Let $\mathcal{I}_{k}$ for all $k \in \mathcal{K}$ be interference functions. Hence, these functions $\mathcal{I}_{k}$, for all $k \in \mathcal{K}$, are strictly monotonic with respect to $p_{K+1}$. Let $\boldsymbol{\gamma}^{(1)}>\mathbf{0}$ and $C\left(\boldsymbol{\gamma}^{(1)}, \mathcal{I}, P_{\text {total }}\right)=1$. Since we are in the case with power constraints (there exists noise), our interference function framework is equivalent to the Yates interference function framework. Then, from the fixed-point theorem in [6], we know that there exists a power vector $\underline{\boldsymbol{p}}^{(1)}>\mathbf{0}$ with $p_{k}^{(1)}=$ $\gamma_{k}^{(1)} \mathcal{I}_{k}\left(\underline{\boldsymbol{p}}^{(1)}\right)$, for $k \in \mathcal{K}$ and $\sum_{k \in \mathcal{K}} p_{k}^{(1)} \leq P_{\text {total }}$. Assume that there exists a SINR vector $\boldsymbol{\gamma}^{(2)} \geq \boldsymbol{\gamma}^{(1)}$ with $\boldsymbol{\gamma}^{(2)} \neq \gamma^{(1)}$ and

$$
C\left(\gamma^{(2)}, \mathcal{I}, P_{\text {total }}\right)=1 \text {. }
$$

From (28), we know that there exists a corresponding power vector $\underline{\boldsymbol{p}}^{(2)}$ such that $\sum_{k \in \mathcal{K}} p_{k}^{(2)} \leq P_{\text {total }}$

$$
\begin{aligned}
p_{k}^{(2)} & =\gamma_{k}^{(2)} \mathcal{I}_{k}\left(\underline{\boldsymbol{p}}^{(2)}\right) & & \forall k \in \mathcal{K} \\
& \geq \gamma_{k}^{(1)} \mathcal{I}_{k}\left(\underline{\boldsymbol{p}}^{(2)}\right) & & k \in \mathcal{K} .
\end{aligned}
$$

The first equality above follows from the fact that $\gamma^{(2)}$ is a boundary point, and the second inequality follows from our choice of SINR vectors as $\boldsymbol{\gamma}^{(2)} \geq \boldsymbol{\gamma}^{(1)}$. We utilize the power vector $\underline{\boldsymbol{p}}^{(2)}$ to construct a new power vector $\underline{\tilde{\boldsymbol{p}}}$ such that $\sum_{k=1}^{K} \tilde{p}_{k}<\bar{P}_{\text {total }}$ and

$$
\tilde{p}_{k} \geq \gamma_{k} \mathcal{I}_{k}(\underline{\tilde{\boldsymbol{p}}}), \quad k \in \mathcal{K}
$$

as follows: We select an index $k_{0}$ such that $\tilde{p}_{k_{0}}<p_{k_{0}}^{(2)}$ and $\tilde{p}_{k_{0}}>\gamma_{k_{0}}^{(1)} \mathcal{I}_{k_{0}}\left(\underline{\boldsymbol{p}}^{(2)}\right)$. Such an index $k_{0}$ always exists since we have selected $\boldsymbol{\gamma}^{(\overline{2})} \geq \boldsymbol{\gamma}^{(1)}$ with $\boldsymbol{\gamma}^{(2)} \neq \boldsymbol{\gamma}^{(1)}$. Hence, the power vector $\underline{\boldsymbol{p}}^{(2)}$ corresponding to $\boldsymbol{\gamma}^{(2)}$ can be strictly varied in at least one component $\left(\boldsymbol{\gamma}^{(2)}\right.$ is greater than $\boldsymbol{\gamma}^{(1)}$ in at least one component). One of these components could be the index $k_{0}$.

We now define for $k \neq k_{0}$ that $\tilde{p}_{k}=p_{k}^{(2)}$ and $\sum_{k \in \mathcal{K}} p_{k}^{(2)} \leq$ $P_{\text {total }}$. This gives us that $\tilde{\boldsymbol{p}} \leq \underline{\boldsymbol{p}}^{(2)}$, and for $k=k_{0}$, we have that

$$
\begin{aligned}
\tilde{p}_{k_{0}} & >\gamma_{k_{0}}^{(1)} \mathcal{I}_{k_{0}}\left(\underline{\boldsymbol{p}}^{(2)}\right) \\
& \geq \gamma_{k_{0}}^{(1)} \mathcal{I}_{k_{0}}(\underline{\tilde{\boldsymbol{p}}}) .
\end{aligned}
$$

Equation (30) implies that $\sum_{k \in \mathcal{K}} \tilde{p}_{k}<P_{\text {total }}$ and

$$
\begin{aligned}
\tilde{p}_{k} & =p_{k}^{(2)} \geq \gamma_{k}^{(1)} \mathcal{I}_{k}\left(\underline{\boldsymbol{p}}^{(2)}\right) \\
& \geq \gamma_{k}^{(1)} \mathcal{I}_{k}(\underline{\tilde{\boldsymbol{p}}}) \quad \text { for } k \neq k_{0} .
\end{aligned}
$$

We can now choose $\underline{\tilde{\boldsymbol{p}}}(\lambda)=\lambda \underline{\tilde{\boldsymbol{p}}}$ with $1<\lambda<P_{\text {total }} / \sum_{k=1}^{K} \tilde{p}_{k}$. We have that $\sum_{k=1}^{K} \tilde{p}_{k}(\lambda)<P_{\text {total }}$ and for $1 \leq k \leq K$

$$
\tilde{p}_{k}(\lambda)=\lambda \tilde{p}_{k}=\lambda \gamma_{k} \mathcal{I}_{k}(\underline{\tilde{\boldsymbol{p}}})>\gamma_{k} \mathcal{I}_{k}\left(\lambda \tilde{\boldsymbol{p}}, \sigma^{2}\right)
$$

where $\underline{\tilde{\boldsymbol{p}}}=\left[\tilde{\boldsymbol{p}}, \sigma^{2}\right]$. In the last step in (31), we are scaling the power vector and not scaling the noise. The last inequality in (31) follows from the choice of the power vector $\tilde{\boldsymbol{p}}$ and from the property of strict monotonicity of the interference function with respect to the noise component (axiom $A 4$; see Definition 8). We can utilize $A 4$ since we are in the case with power constraints. Equation (31) gives us that $C\left(\gamma^{(1)}, \mathcal{I}, P_{\text {total }}\right)<1$, giving us the required contradiction.

Example 4: Consider a two-user Gaussian broadcast channel (BC) with dirty paper coding normalized noise $\sigma_{n}^{2}=1$. The SINRs of the users $\gamma=\left[\gamma_{1}, \gamma_{2}\right]^{T}$ are $\gamma_{1}=\frac{p_{1}}{p_{2}+1}$ 


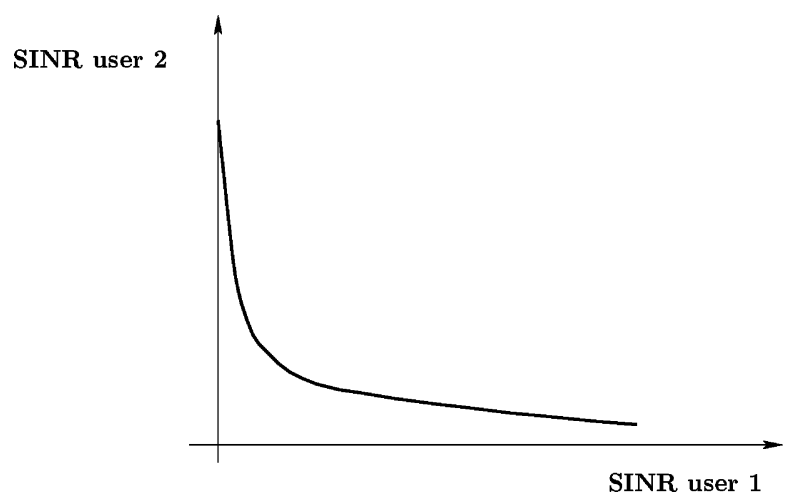

Fig. 5. SINR feasible region for the two-user $\mathrm{BC}$ channel described in Example $4, P_{\text {total }}=2$.

and $\gamma_{2}=p_{2}$. Then, we have that the powers of the individual users are $p_{1}=\gamma_{1}\left(p_{2}+1\right)=\gamma_{1}\left(\gamma_{2}+1\right)$ and $p_{2}=\gamma_{2}$. Assuming a total power constraint $p_{1}+p_{2} \leq P_{\text {total }}$, we obtain the SINR region as depicted in Fig. 5. We have that the SINR of user 2 is given by $\gamma_{2}=\frac{P_{\text {total }}+\gamma_{1}}{1+\gamma_{1}}$

The characterization of the boundary points of feasible utility sets for the special case of log-convex interference functions is discussed in [25]. Next, we characterize the structure of the region, specifically the boundary points of the region with respect to the behavior of the interference-balancing functions $C(\boldsymbol{\gamma}, \mathcal{I})$ and $C\left(\boldsymbol{\gamma}, \mathcal{I}, \boldsymbol{p}^{\max }\right)$. We would like to segregate feasible utility regions based on whether they have Pareto-optimal or weak Pareto-optimal boundary points. We are aware that the functions $C(\boldsymbol{\gamma}, \mathcal{I})$ and $C\left(\boldsymbol{\gamma}, \mathcal{I}, \boldsymbol{p}^{\max }\right)$ are interference functions with respect to $\gamma_{1}, \ldots, \gamma_{K}$, i.e., they satisfy axioms $A 1, A 2$ and $A 3$.

\section{Characterization OF BOUNDARY POINTS With RESPECT TO BEHAVIOR OF THE INTERFERENCE-BALANCING FUNCTIONS}

So far, we have characterized the Pareto optimality of the boundary points of utility sets with respect to certain properties of interference functions. In this section, we present the connection between the behavior of boundary points and the interference-balancing functions ( $C$ functions) for SIR vectors $\boldsymbol{\gamma}>\mathbf{0}$. These results complement the analysis by providing an alternative means of characterizing boundary points, namely via the properties of the interference-balancing functions.

Theorem 5: All boundary points of feasible utility sets without power constraints are Pareto-optimal if and only if the corresponding interference-balancing function $C(\boldsymbol{\gamma}, \mathcal{I})$ is a strictly monotonic function on $\mathbb{R}_{++}^{K}$.

All boundary points of feasible utility sets with individual power constraints are Pareto-optimal if and only if the corresponding interference-balancing function $C\left(\boldsymbol{\gamma}, \mathcal{I}, \boldsymbol{p}^{\max }\right)$ is a strictly monotonic function on $\mathbb{R}_{++}^{K}$.

Proof: If the function $C(\boldsymbol{\gamma}, \mathcal{I})$ is strictly monotonic, then all boundary points $\boldsymbol{\gamma}$ (due to the condition $C(\boldsymbol{\gamma}, \mathcal{I})=1$ ) are Pareto-optimal.

Conversely, for the sake of contradiction, assume that $C(\boldsymbol{\gamma}, \mathcal{I})$ is not strictly monotonic and all boundary points are Paretooptimal. Choose two SIR vectors $\boldsymbol{\gamma}^{(1)}, \boldsymbol{\gamma}^{(2)}$ such that $\boldsymbol{\gamma}^{(1)} \geq$ $\boldsymbol{\gamma}^{(2)}$ and $\boldsymbol{\gamma}^{(1)} \neq \boldsymbol{\gamma}^{(2)}$ with $\mathbf{0}<C\left(\boldsymbol{\gamma}^{(1)}, \mathcal{I}\right)=C\left(\boldsymbol{\gamma}^{(2)}, \mathcal{I}\right)$ (they exist since $C$ is not a strictly monotonic function). Now choose $\hat{\lambda} \boldsymbol{\gamma}^{(1)}, \hat{\lambda} \boldsymbol{\gamma}^{(2)}$ and $\lambda>0$ such that $C\left(\hat{\lambda} \boldsymbol{\gamma}^{(1)}, \mathcal{I}\right)=1$, i.e., $\hat{\lambda}=\frac{1}{C\left(\boldsymbol{\gamma}^{(1)} \mathcal{I}\right)}$. Then, $\hat{\boldsymbol{\gamma}}^{(1)}=\lambda \boldsymbol{\gamma}^{(1)}$ and $\hat{\boldsymbol{\gamma}}^{(2)}=\lambda \boldsymbol{\gamma}^{(2)}$ are boundary points with $\hat{\boldsymbol{\gamma}}^{(1)} \geq \hat{\boldsymbol{\gamma}}^{(2)}$ and $\hat{\boldsymbol{\gamma}}^{(1)} \neq \hat{\boldsymbol{\gamma}}^{(2)}$. Furthermore, $C\left(\gamma^{(1)}, \mathcal{I}\right)=C\left(\boldsymbol{\gamma}^{(2)}, \mathcal{I}\right)$, i.e., $\hat{\gamma}^{(2)}$ is not Pareto-optimal. We have our desired contradiction, which proves our result that if all boundary points are Pareto-optimal, then the function $C(\boldsymbol{\gamma}, \mathcal{I})$ is strictly monotonic.

Similar arguments could be used to prove the result for the case of individual power constraints with strictly monotonic $C\left(\boldsymbol{\gamma}, \mathcal{I}, \boldsymbol{p}^{\max }\right)$ functions.

It can be observed that the proof of Theorem 5 was quite straightforward, and we have displayed that there is a simple connection between the monotonic behavior of the $C(\boldsymbol{\gamma}, \mathcal{I})$ function and the Pareto-optimal boundary points. Now, we can utilize Theorem 2 to completely describe all possible cases when the function $C(\gamma, \mathcal{I})$ defined by (6) is strictly monotonic.

Corollary 1: Let $\mathcal{I}_{1}, \ldots, \mathcal{I}_{K}$ be strictly positive interference functions on their respective dependency sets. Let $\mathcal{I}_{1}, \ldots \mathcal{I}_{K}$ be strictly monotonic on their respective dependency sets. The interference-balancing function $C(\gamma, \mathcal{I})$ for the case without power constraints is strictly monotonic if and only if the global dependency matrix $\boldsymbol{G}$ is irreducible.

Proof: The proof is a direct application of Theorems 2 and 5.

We assume that interference functions $\mathcal{I}_{k}$ for all $k \in \mathcal{K}$ in (6) are strictly monotonic on their respective dependency sets. Corollary 1 displays the conditions on "user coupling" such that we have strict monotonicity of the $C$ function for all variables.

Corollary 2: Let $\mathcal{I}_{1}, \ldots, \mathcal{I}_{K}$ be strictly positive interference functions on their respective dependency sets. Let $\mathcal{I}_{1}, \ldots, \mathcal{I}_{K}$ be strictly monotonic on their respective dependency sets. The interference-balancing function $C\left(\boldsymbol{\gamma}, \mathcal{I}, \boldsymbol{p}^{\max }\right)$ for the case with individual power constraints is strictly monotonic if and only if the global dependency matrix $\boldsymbol{G}$ is irreducible.

Proof: The proof is a direct application of Theorems 2 and 5.

Corollaries 1 and 2 describe the relationship between the interference-balancing functions and the global dependency matrix $\boldsymbol{G}$. As can be seen, we have an interplay between certain properties of the interference-balancing functions, the combinatorial characteristics of the global dependency matrix and the Pareto optimality of the boundary points of the feasible utility regions.

\section{CONCLUSION}

We have analyzed the structure of certain utility sets without power constraints and with power constraints. The connection between Pareto optimality and interference coupling has been displayed. We have introduced an intuitive constraint in wireless systems (with mathematical implications), namely that of strict positivity of the interference functions on the dependency set. It was shown that boundary points of utility sets without power constraints with strictly positive interference functions on their respective dependency sets and strictly monotonic interference functions on their respective dependency sets are 
Pareto-optimal if and only if the corresponding global dependency matrix is irreducible. It was shown that the boundary points of utility sets with individual power constraints and interference functions, which are strictly monotonic on their respective dependency sets, are Pareto-optimal if and only if the corresponding restricted global dependency matrix is irreducible. It was shown that the boundary points of utility sets with a total power constraint for arbitrary interference functions are Pareto-optimal. The relation between interference-balancing functions ( $C$ functions) and Pareto optimality of the boundary points was elucidated. We have provided a complete characterization of the Pareto optimality of the boundary points of feasible utility sets, which can be used as a building block to investigate other complex problems dependent on this property of the boundary points.

\section{APPENDIX A}

PROOF OF LEMMA 3

Proof: We shall achieve this proof by contradiction. Let $\gamma_{1}, \ldots, \gamma_{K}>0$ be such that $C(\gamma, \mathcal{I})=0$. Then, for all $\epsilon>0$, there exists a vector $\boldsymbol{p}(\epsilon), \boldsymbol{p}(\epsilon)>$ $\mathbf{0},\|\boldsymbol{p}\|_{\infty}=1$ and $\left(\gamma_{k} \mathcal{I}_{k}(\boldsymbol{p}(\epsilon))\right) / p_{k}(\epsilon)<\epsilon, 1 \leq k \leq K$, i.e., $\gamma_{k} \mathcal{I}_{k}(\boldsymbol{p}(\epsilon))<\epsilon p_{k}(\epsilon)$. Let $l_{0}$ be an index with $p_{l_{0}}$ with $p_{l_{0}}(\epsilon)=1$. Let $k_{0}$ be an index such that $\mathcal{I}_{k_{0}}$ is an interference function with $l_{0} \in L_{k_{0}}$. Then, we have that $0<c_{3}:=\gamma_{k_{0}} \mathcal{I}_{k_{0}}\left(\boldsymbol{e}\left(l_{0}\right)\right) \leq \gamma_{k_{0}} \mathcal{I}_{k_{0}}(\boldsymbol{p}(\epsilon))<\epsilon p_{k_{0}}(\epsilon)=\epsilon$. Therefore, for all $\epsilon>0$, we must have that $0<c_{3} \leq \epsilon$ and $c_{3}>0$, which gives us our required contradiction.

\section{APPENDIX B}

\section{PROOF OF LEMMA 4}

Proof: We have that the interference-balancing function $C(\boldsymbol{\gamma}, \mathcal{I})$ can be written as follows: $C(\boldsymbol{\gamma}, \mathcal{I})=$ $\inf _{\boldsymbol{p}>\mathbf{0}} \max _{k \in \mathcal{K}} \frac{\gamma_{k} \mathcal{I}_{k}(\boldsymbol{p})}{p_{k}} \geq C\left(\boldsymbol{\gamma}^{(l)}, \mathcal{I}^{(l)}\right)$ for $l=\{1,2\}$. We consider the following two cases.

Case (i): $C\left(\boldsymbol{\gamma}^{(1)}, \mathcal{I}^{(1)}\right) \geq C\left(\boldsymbol{\gamma}^{(2)}, \mathcal{I}^{(2)}\right)$ : For all $\epsilon>0$ we can find a power vector $\boldsymbol{p}^{(1)}(\epsilon) \in \mathbb{R}_{++}^{k_{1}}$ and another power vector $\boldsymbol{p}^{(2)}(\epsilon) \in \mathbb{R}_{+, 0}^{K, k_{1}}$, such that

$$
\begin{aligned}
& \frac{\gamma_{k} \mathcal{I}_{k}\left(\boldsymbol{p}^{(1)}(\epsilon)\right)}{p_{k}^{(1)}(\epsilon)}<C\left(\boldsymbol{\gamma}^{(1)}, \mathcal{I}^{(1)}\right)+\epsilon, \quad 1 \leq k \leq k_{1} \\
& \frac{\gamma_{k} \mathcal{I}_{k}\left(\boldsymbol{p}^{(2)}(\epsilon)\right)}{p_{k}^{(2)}(\epsilon)} \stackrel{\text { (b) }}{<} C\left(\boldsymbol{\gamma}^{(2)}, \mathcal{I}^{(2)}\right)+\epsilon, \quad k_{1}<k \leq K .
\end{aligned}
$$

We choose a power vector $\tilde{\boldsymbol{p}}(\mu)$ such that $\tilde{\boldsymbol{p}}(\mu)=$ $\left[\boldsymbol{p}^{(1)}(\epsilon), \mathbf{0}_{k_{1}+1, K}\right]^{T}+\boldsymbol{\mu} \boldsymbol{p}^{(2)}(\epsilon)$, where $\mathbf{0}_{k_{1}+1, K}$ is a vector of zeros of dimensions $K-k_{1}$ and $\mu>1$. We have for $k_{1}+1 \leq k \leq K$ that

$$
\lim _{\mu \rightarrow \infty} \frac{\mathcal{I}_{k}(\tilde{\boldsymbol{p}}(\mu))}{\tilde{p}_{k}(\mu)}=\frac{\mathcal{I}_{k}\left(\boldsymbol{p}^{(2)}(\epsilon)\right)}{p_{k}^{(2)}(\epsilon)} .
$$

From (b), there exists a scalar $\tilde{\mu}$ such that

$$
\frac{\gamma_{k} \mathcal{I}_{k}(\tilde{\boldsymbol{p}}(\tilde{\mu}))}{\tilde{p}_{k}(\tilde{\mu})}<C\left(\boldsymbol{\gamma}^{(2)}, \mathcal{I}^{(2)}\right)+\epsilon
$$

for all $k_{1}+1 \leq k \leq K$.

Furthermore, $\mathcal{I}_{k}\left(\boldsymbol{p}^{(1)}(\epsilon)\right)=\mathcal{I}_{k}(\tilde{\boldsymbol{p}}(\tilde{\mu}))$, for $1 \leq k \leq k_{1}$. Therefore, we have that

$$
\begin{aligned}
C(\boldsymbol{\gamma}, \mathcal{I}) & =\inf _{\boldsymbol{p} \in \mathbb{R}_{++}^{K}} \max _{k \in \mathcal{K}} \frac{\gamma_{k} \mathcal{I}_{k}(\boldsymbol{p})}{p_{k}} \\
& \leq \max _{k \in \mathcal{K}} \frac{\gamma_{k} \mathcal{I}_{k}(\tilde{\boldsymbol{p}}(\tilde{\mu}))}{\tilde{p}_{k}(\tilde{\mu})} \\
& \leq C\left(\boldsymbol{\gamma}^{(1)}, \mathcal{I}^{(1)}\right)+\epsilon \quad \forall \epsilon>0 .
\end{aligned}
$$

Then, we have that $C(\boldsymbol{\gamma}, \mathcal{I})=C\left(\boldsymbol{\gamma}^{(1)}, \mathcal{I}^{(1)}\right)$, which is the desired result.

Case (ii): $C\left(\boldsymbol{\gamma}^{(1)}, \mathcal{I}^{(1)}\right)<C\left(\boldsymbol{\gamma}^{(2)}, \mathcal{I}^{(2)}\right)$ : This case can be analyzed in a similar fashion as the previous case. We now prove the second part of the lemma. Let us assume that we always have $C\left(\boldsymbol{\gamma}^{(1)}, \mathcal{I}^{(1)}\right)<C(\boldsymbol{\gamma}, \mathcal{I})$ for all SIR vectors $\boldsymbol{\gamma}>\mathbf{0}$. Then, we have that

$$
C(\boldsymbol{\gamma}, \mathcal{I})=C\left(\boldsymbol{\gamma}^{(2)}, \mathcal{I}^{(2)}\right) .
$$

Now, we choose an arbitrary SIR vector $\boldsymbol{\gamma}>\boldsymbol{0}$. Then, we have that $C\left(\boldsymbol{\gamma}^{(1)}, \mathcal{I}^{(1)}\right)=: c_{1}>0$. We choose $\gamma_{k}(\lambda)=\lambda \gamma_{k}$, for all $1 \leq k \leq k_{1}$, and $\gamma_{k}(\lambda)=\gamma_{k}$, for all $k_{1}+1 \leq k \leq$ $K$. Then, we have that for all $\lambda \geq 1$ by assumption (32) that $C(\gamma(\lambda), \mathcal{I})=C\left(\boldsymbol{\gamma}^{(2)}(\lambda), \mathcal{I}^{(2)}\right)=C\left(\boldsymbol{\gamma}^{(2)}, \mathcal{I}^{(2)}\right)=: c_{2}$. We are aware that $C\left(\boldsymbol{\gamma}^{(1)}(\lambda), \mathcal{I}^{(1)}\right)=\lambda C\left(\boldsymbol{\gamma}^{(1)}, \mathcal{I}^{(1)}\right)=\lambda c_{1}$. We now have that $\lambda c_{1}<\lambda c_{2}$, for all $\lambda>1$. We now have our desired contradiction since we have that $c_{1}>0$.

\section{APPENDIX C \\ INTERFERENCE FUNCTION FRAMEWORK}

Let $\mathcal{P}$ be the set of all power vectors. In our paper, we have $\mathcal{P}:=\mathbb{R}_{+}^{K+1}$ unless explicitly mentioned otherwise.

Definition 8: We say that $\mathcal{I}: \mathcal{P} \mapsto \mathbb{R}_{+}$is an interference function if the following axioms are fulfilled:

$$
\begin{array}{ll}
A 1 & \text { conditional positivity } \mathcal{I}(\underline{\boldsymbol{p}})>0 \text { if } \underline{\boldsymbol{p}}>\mathbf{0} \\
A 2 & \text { scale invariance } \mathcal{I}(\alpha \underline{\boldsymbol{p}})=\alpha \mathcal{I}(\underline{\boldsymbol{p}}) \text { for all } \alpha>0 \\
A 3 & \text { monotonicity } \mathcal{I}(\underline{\boldsymbol{p}}) \geq \mathcal{I}(\underline{\hat{\boldsymbol{p}}}) \text { if } \underline{\boldsymbol{p}} \geq \underline{\hat{\boldsymbol{p}}} \\
A 4 & \text { strict monotonicity } \mathcal{I}(\underline{\boldsymbol{p}})>\mathcal{I}(\underline{\hat{\boldsymbol{p}}}) \text { if } \underline{\boldsymbol{p}} \geq \underline{\hat{\boldsymbol{p}}}
\end{array}
$$

$$
p_{K+1}>\hat{p}_{K+1} .
$$

Note that we require that $\mathcal{I}(\boldsymbol{p})$ is strictly monotonic with respect to the last component $p_{K+1}$. An example is $\mathcal{I}(\underline{p})=\boldsymbol{v}^{T} \boldsymbol{p}+$ $\sigma^{2}$, where $\boldsymbol{v} \in \mathbb{R}_{+}^{K}$ is a vector of interference coupling coefficients. The axiomatic framework A1-A4 is connected with the framework of standard interference functions [6]. For any constant noise power $p_{K+1}=\sigma^{2}$, the function $Y(\boldsymbol{p})=\mathcal{I}(\boldsymbol{p})$ is standard. Conversely, every standard interference function can be expressed within the framework A1-A4. Let $Y$ be a standard interference function, then $\mathcal{I}(\underline{\boldsymbol{p}})=p_{K+1} \cdot Y\left(\frac{p_{1}}{p_{K+1}}, \ldots, \frac{p_{K}}{p_{K+1}}\right)$ 
is an interference function fulfilling A1-A4. We have $Y(\boldsymbol{p})=$ $\mathcal{I}(\underline{p})$ for all $\boldsymbol{p}>\mathbf{0}, p_{K+1}=1$. The details about the relationship between the model A1-A4 and Yates' standard interference functions were discussed in [11] and further investigated in [16]. For the purpose of this paper, it is sufficient to be aware that there exists a connection between these two models, and the results of this paper are applicable to standard interference functions.

\section{REFERENCES}

[1] H. J. M. Peters, Axiomatic Bargaining Game Theory. Norwell, MA: Kluwer, 1992.

[2] L. Hurwicz, "On informationally decentralized systems," in Decision and Organization. Amsterdam, The Netherlands: North-Holland, 1972, pp. 297-336.

[3] L. Zhou, "Inefficiency of strategy-proof allocation mechanisms in pure exchange economies," Social Choice Welfare, vol. 8, pp. 247-254, 1991.

[4] H. Boche and S. Stanczak, "Strict convexity of the feasible log-SIR region," IEEE Trans. Commun., vol. 56, no. 9, pp. 1511-1518, Sep. 2008.

[5] H. Boche and M. Schubert, "Nash bargaining and proportional fairness for wireless systems," IEEE/ACM Trans. Netw., vol. 17, no. 5, pp. 1453-1466, Oct. 2009.

[6] R. D. Yates, "A framework for uplink power control in cellular radio systems," IEEE J. Sel. Areas Commun., vol. 13, no. 7, pp. 1341-1348, Sep. 1995.

[7] C. Huang and R. Yates, "Rate of convergence for minimum power assignment algorithms in cellular radio systems," Wireless Netw., vol. 4, pp. 223-231, 1998.

[8] K. K. Leung, C. W. Sung, W. S. Wong, and T. Lok, "Convergence theorem for a general class of power-control algorithms," IEEE Trans. Commun., vol. 52, no. 9, pp. 1566-1574, Sep. 2004.

[9] M. Bengtsson and B. Ottersten, "Optimal and suboptimal transmit beamforming," in Handbook of Antennas in Wireless Communications. Boca Raton, FL: CRC Press, Aug. 2001, ch. 18.

[10] S. Ulukus and R. Yates, "Adaptive power control and MMSE interference suppression," Wireless Netw., vol. 4, no. 6, pp. 489-496, 1998.

[11] M. Schubert and H. Boche, "QoS-based resource allocation and transceiver optimization," Found. Trends Commun. Inf. Theory, vol. 2, no. 6, pp. 383-529, 2005/2006.

[12] H. Boche and M. Schubert, "A superlinearly and globally convergent algorithm for power control and resource allocation with general interference functions," IEEE/ACM Trans. Netw., vol. 16, no. 2, pp. 383-395, Apr. 2008.

[13] N. Bambos, S. Chen, and G. Pottie, "Channel access algorithms with active link protection for wireless communication networks with power control," IEEE/ACM Trans. Netw., vol. 8, no. 5, pp. 583-597, Oct. 2000.

[14] M. Xiao, N. Shroff, and E. Chong, "A utility-based power- control scheme in wireless cellular systems," IEEE/ACM Trans. Netw., vol. 11, no. 2, pp. 210-221, Apr. 2003.

[15] A. Koskie and Z. Gajic, "A Nash game algorithm for SIR-based power control for 3G wireless CDMA networks," IEEE/ACM Trans. Netw., vol. 13, no. 5, pp. 1017-1026, Oct. 2005.

[16] H. Boche and M. Schubert, "A unifying approach to interference modeling for wireless networks," IEEE Trans. Signal Process., vol. 58, no. 6, pp. 3282-3297, Jun. 2010.

[17] H. Boche and M. Schubert, "A generalization of Nash bargaining and proportional fairness to log-convex utility sets with power constraints," IEEE Trans. Inf. Theory, 2010, accepted for publication.

[18] R. Horn and C. Johnson, Matrix Analysis. Cambridge, U.K.: Cambridge Univ. Press, 1985.

[19] E. Seneta, Non-Negative Matrices and Markov Chains. Berlin, Germany: Springer, 1981

[20] J. Zander and S. L. Kim, Radio Resource Management for Wireless Networks. Boston, MA: Artech House, 2001.

[21] M. Schubert and H. Boche, "A generic approach to QoS-based transceiver optimization," IEEE Trans. Commun., vol. 55, no. 8, pp. 1557-1566, Aug. 2007.
[22] E. Maskin, "Nash equilibrium and welfare optimality," Rev. Econ Studies, vol. 66, pp. 23-38, 1999.

[23] W. Rudin, Real and Complex Analysis, 2nd ed. New York: McGrawHill, 1974.

[24] F. R. Gantmacher, The Theory of Matrices. New York: Chelsea, 1959, vol. 2.

[25] H. Boche and M. Schubert, "Complete characterization of the Pareto boundary of interference coupled wireless systems with power constraints-The log-convex case," in Proc. IEEE ICASSP, Taipei, Taiwan, Apr. 2009, pp. 3637-3640.

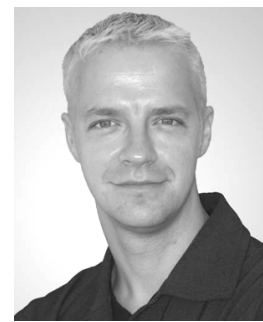

Holger Boche (M'04-SM'07) received the Dipl.-Ing. and Dr.-Ing. degrees in electrical engineering from the Technische Universitaet Dresden, Dresden, Germany, in 1990 and 1994, respectively. He graduated in mathematics from the Technische Universitaet Dresden in 1992. From 1994 to 1997 , he did postgraduate studies in mathematics at the Friedrich-Schiller Universität Jena, Jena, Germany. He received his Dr.Rer.Nat. degree in pure mathematics from the Technische Universitaet Berlin, Berlin, Germany, in 1998.

In 1997, he joined the Heinrich-Hertz-Institut (HHI) für Nachrichtentechnik Berlin, Berlin, Germany. Since 2002, he has been a Full Professor for mobile communication networks with the Institute for Communications Systems, Technische Universität Berlin. In 2003, he became Director of the Fraunhofer German-Sino Lab for Mobile Communications, Berlin, Germany, and since 2004 he has also been Director of the Fraunhofer Institute for Telecommunications (HHI), Berlin, Germany. He was a Visiting Professor with the ETH Zurich, Zurich, Switzerland, during the 2004 and 2006 Winter terms, and with KTH Stockholm, Stockholm, Sweden, during the 2005 Summer term.

Prof. Boche is a Member of IEEE Signal Processing Society SPCOM and SPTM Technical Committee. He was elected a Member of the German Academy of Sciences (Leopoldina) in 2008 and of the Berlin Brandenburg Academy of Sciences and Humanities in 2009. He received the Research Award "Technische Kommunikation" from the Alcatel SEL Foundation in October 2003, the "Innovation Award" from the Vodafone Foundation in June 2006, and the Gottfried Wilhelm Leibniz Prize from the Deutsche Forschungsgemeinschaft (German Research Foundation) in 2008. He was co-recipient of the 2006 IEEE Signal Processing Society Best Paper Award and recipient of the 2007 IEEE Signal Processing Society Best Paper Award.

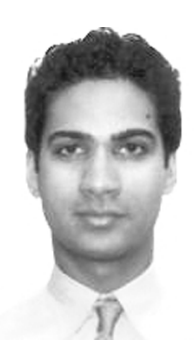

Siddharth Naik received the M.S. degree in electrical engineering from the Royal Institute of Technology, Stockholm, Sweden, in 2004 and is currently pursuing the $\mathrm{Ph} . \mathrm{D}$. degree in electrical engineering at the Technical University of Berlin, Berlin, Germany.

He was a Research Engineer with the SPANN laboratory, Indian Institute of Technology, Mumbai, India, in 2004, and a Strategy Consultant in 2005 . $\mathrm{He}$ has been a Research Associate with the Heinrich-Hertz Institute, Berlin, Germany, since 2006 His research interests are game theory, mechanism design, probability theory, and nonlinear optimization.

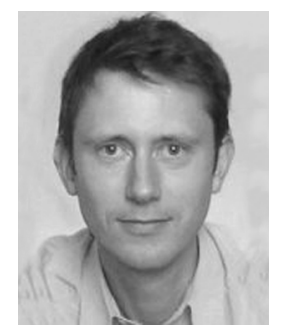

Martin Schubert (M'06) received the diploma and doctoral degrees in electrical engineering from the Technische Universität, Berlin, Germany, in 1998 and 2002, respectively.

In 1998, he joined the Heinrich-Hertz Institute for Telecommunications (HHI), Berlin, Germany, as a Research Assistant. Since 2003, he has been with the Fraunhofer German-Sino Lab for Mobile Communications (MCI), where he is working as a Senior Researcher. He is also a Lecturer with the Technical University of Berlin.

Dr. Schubert has been an Associate Editor of the IEEE TRANSACTIONS OF SigNAL PROCESSING since 2009. He was a co-recipient of the VDE JohannPhilipp-Reis Award in 2007. He coauthored the 2007 Best Paper Award of the IEEE Signal Processing Society. 